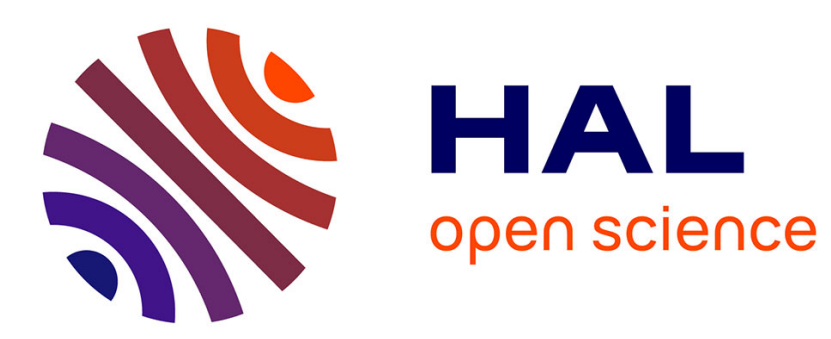

\title{
On bandwidth selection problems in nonparametric trend estimation under martingale difference errors
}

Karim Benhenni, Didier A. Girard, Sana Louhichi

\section{To cite this version:}

Karim Benhenni, Didier A. Girard, Sana Louhichi. On bandwidth selection problems in nonparametric trend estimation under martingale difference errors. Bernoulli, 2022, 28 (1), pp.395-423. 10.3150/21BEJ1347 . hal-02514827v2

\section{HAL Id: hal-02514827 \\ https://hal.science/hal-02514827v2}

Submitted on 10 Apr 2021

HAL is a multi-disciplinary open access archive for the deposit and dissemination of scientific research documents, whether they are published or not. The documents may come from teaching and research institutions in France or abroad, or from public or private research centers.
L'archive ouverte pluridisciplinaire HAL, est destinée au dépôt et à la diffusion de documents scientifiques de niveau recherche, publiés ou non, émanant des établissements d'enseignement et de recherche français ou étrangers, des laboratoires publics ou privés. 


\title{
On bandwidth selection problems in nonparametric trend estimation under martingale difference errors
}

\author{
Karim Benhenni*, Didier A. Girard† Sana Louhichi ${ }^{\ddagger}$
}

April 10, 2021

\begin{abstract}
In this paper, we are interested in the problem of smoothing parameter selection in nonparametric curve estimation under dependent errors. We focus on kernel estimation and the case when the errors form a general stationary sequence of martingale difference random variables where neither linearity assumption nor "all moments are finite" are required. We compare the behaviors of the smoothing bandwidths obtained by minimizing either the unknown average squared error, the theoretical mean average squared error, a Mallows-type criterion adapted to the dependent case and the family of criteria known as generalized cross validation (GCV) extensions of the Mallows' criterion. We prove that these three minimizers and those based on the GCV family are first-order equivalent in probability. We give also a normal asymptotic behavior of the gap between the minimizer of the average square error and that of the Mallows-type criterion. This is extended to the GCV family. Finally, we apply our theoretical results to a specific case of martingale difference sequence, namely the Auto-Regressive Conditional Heteroscedastic $(\mathrm{ARCH}(1))$ process. A Monte-carlo simulation study, for this regression model with $\mathrm{ARCH}(1)$ process, is conducted.
\end{abstract}

*Laboratoire Jean Kuntzmann, Université Grenoble Alpes, 700 Avenue Centrale, 38401 Saint-Martin-d'Hères, France. E.mail: karim.benhenni@univ-grenoble-alpes.fr

${ }^{\dagger}$ CNRS, Laboratoire Jean Kuntzmann. E.mail: didier.girard@univ-grenoble-alpes.fr

‡Laboratoire Jean Kuntzmann, Université Grenoble Alpes, 700 Avenue Centrale, 38401 Saint-Martin-d'Hères, France. E.mail: sana.louhichi@univ-grenoble-alpes.fr. 
Keywords: Nonparametric trend estimation, Kernel nonparametric models, Smoothing parameter selection, Martingale difference sequences, Average squared error, Mean average squared error, Mallows criterion, Cross validation, Generalized cross validation, $\operatorname{ARCH}(1)$.

2010 Mathematics Subject Classification. 62G08. 62G20. 60G10

\section{Contents}

1 Introduction 2

2 Model and notations $\quad 5$

3 Main results and applications $\quad 9$

3.1 Application to $\mathrm{ARCH}(1)$ processes . . . . . . . . . . . . . 11

3.2 A Monte-carlo simulation study for a "trend plus $\mathrm{ARCH}(1)$ process" ......................... 13

4 Proofs $\quad 18$

4.1 Main lemmas for the proof of Proposition 3.1 . . . . . . . . 18

4.1.1 Proof of Lemma 4.1 . . . . . . . . . . . . . . . . 19

4.1.2 Proof of Lemma 4.2 . . . . . . . . . . . . . 23

4.2 Proof of Proposition 3.1 . . . . . . . . . . . . . . . 25

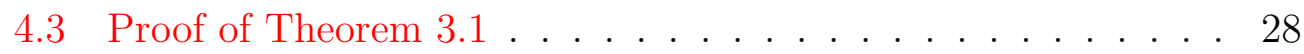

4.3.1 Step 1: convergence in distribution of $a_{n} \delta_{2}^{\prime}\left(h_{n}\right)$. . . 29

4.3.2 Step 2: convergence in probability of $a_{n}\left(\delta_{2}^{\prime}\left(h_{n}\right)-\delta_{2}^{\prime}\left(\hat{h}_{n}\right)\right) 31$

4.3.3 Step 3: convergence in probability of $\frac{\mathrm{CL}^{\prime \prime}\left(h^{*}\right)}{\mathbb{E}\left(T_{\prime \prime}^{\prime \prime}\left(h_{n}\right)\right)} \ldots . .32$

4.3.4 Step 4: uniform convergence in probability, over $H_{n}$, of $a_{n}\left(\delta_{3}^{\prime}(h)-\delta_{2}^{\prime}(h)\right)$, where $\delta_{3}$ is defined in Lemma 4.3 .34

4.3.5 Step 5: convergence in probability of $\frac{G_{\mathrm{X}}^{\prime \prime}\left(h^{*}\right)}{\mathbb{E}\left(T_{n}^{\prime \prime}\left(h_{n}\right)\right)} \ldots . .35$

4.3.6 End of the proof of Theorem 3.1 . . . . . . . . 35

\section{Introduction}

This paper is about nonparametric regression model (known also as a machine learning function) which is used as a tool to describe and to analyse the trend between a response variable and one or more explanatory random variables. This subject was studied by several authors since 1964 (Nadaraya, 
E. A. (1964), Watson, G. S. (1964)) and is still relevant, due to the fact that nonparametric regression has a lot of applications in different fields, such as economics, medicine, biology, physics, environment, social sciences, $\cdots$, see for instance Hastie, T., Tibshirani, R. and Friedman, J. (2009).

Several estimate of the nonparametric regression function are proposed in the literature such as kernel smoothing, local polynomial regression, splinebased regression models, and regression trees (see for instance Hastie, T., Tibshirani, R. and Friedman, J. (2009)). In this paper, we are interested in kernel nonparametric estimations. These estimate depend on some smoothing parameter $h$ which has to be chosen according to some criteria. For independent observations, two popular criteria, to select $h$, are known as the Cross Validation (CV) criterion and its rotation-invariant version called Generalized Cross-Validation (GCV) criterion. The GCV criterion has different variants, see for instance Akaike, H. (1970), Craven, P. and Wahba, G. (1979), Shibata, R. (1981), Rice, J. (1984), Mallows, C. L. (1973). We refer the reader to Härdle, W., Hall, P. and Marron, J. S. (1988) who studied this problem in the case of independent, equally spaced, observations. They gave, in particular, the behaviors of the minimizers over $h$ of the average squared errors, the mean average squared errors, the cross-validation score CV or the generalized cross-validation GCV. They also studied the deviation between these selected smoothing parameters.

In many cases, independence of the observations is, however, not a realistic modeling of observed data. Autoregressive models, autoregressive conditional heteroscedasticity models, Markov chains are examples of dependent models (see for instance Doukhan, P. and Louhichi, S. (1999)). We focus, in this paper, on the case of kernel nonparametric models with particular dependent errors, more precisely, the case when the errors form a stationary martingale difference sequence (MDS, in short). They are, essentially, two reasons that motivated us to restrict our study of dependence to the case of stationary MDS.

- The first reason is that, studying MDS is a promising step for studying the general case of stationary dependent errors. In fact, MDS plays an important role in establishing the results for arbitrary stationary sequences, see for instance Peligrad, M., Utev, S. and Wu, W. B. (2007) (for moment inequalities purpose).

- The second reason is that MDS is not an abstract notion. Indeed, there are a lot of well known stationary MDS models which are used 
in practice, such as $\mathrm{ARCH}(1)$ or more general $\mathrm{GARCH}(1,1)$ stochastic volatility models.

We compare, in the case of nonparametric regression model with MDS errors, the behaviors of the smoothing bandwidths obtained by minimizing either the unknown average squared error, the theoretical mean average squared error, a Mallows-type criterion adapted to the dependent case and the family of criteria known as generalized cross validation (GCV) extensions of the Mallows' criterion. We prove that these three minimizers and those based on the GCV family are first-order equivalent in probability. We give also a normal asymptotic behavior of the gap between the minimizer of the average square error and that of the Mallows-type criterion. This is extended to the GCV family. The obtained results generalize those under independent errors, as in Härdle, W., Hall, P. and Marron, J. S. (1988), to MDS ones. Finally, we apply our results to a specific case of MDS namely the $\mathrm{ARCH}(1)$ processes.

The adaptation to the dependent case from the independent one is not trivial and needs to establish more theoretical and technical results such as maximal inequalities or limit theorems for quadratic forms of dependent data. To establish our theoretical results, we make use of some ingredients adapted to our case of dependent observations taken from Burkholder, D. L. (1988), Doukhan, P. and Louhichi, S. (1999), McLeish, D. L. (1974) and Rio, E. (1993). Those ingredients are stated in Appendices B and C of the supplementary material. Their proofs are based, in particular, on Marcinkiewicz-Zygmund type inequalities or maximal moment inequalities for MDS, weighted sums of MDS or quadratic forms for MDS that we establish using Burkholder-type moment inequalities together with some chaining arguments (see Lemma C.1., ․ Lemma C.4 and Theorem C.1., Corollary C.1. and Proposition C.1. of Appendix C). Recall that chaining is a nice approach to approximate the supremum, over a non countable set, of stochastic processes (used in the theory of empirical processes see for instance Andrews, D. W. K. and Pollard, D. (1994), Louhichi, S. (2000), or Pollard, D. (1990)). A central limit theorem for triangular arrays of quadratic forms for MDS is also needed for the proofs of our results. We prove this central limit theorem, in Appendix B, by checking the technical conditions of McLeish, D. L. (1974).

Our paper is organized as follows. In Section 2, we introduce the regression model and the different criteria for the selection of the smoothing pa- 
rameter $h$. In Section 3, we state our main results. We apply our theoretical results, in Subsection 3.1, to $\mathrm{ARCH}(1)$ processes. A Monte-carlo simulation study is conducted in Subsection 3.2. An essential part of the proofs of our results are givne in Section 4. A supplementary material Benhenni, Girard and Louhichi (2021) is provided containing the rest of the proofs required for the results of this paper as well as some technical ingredients (see section Supplement).

\section{Model and notations}

Let $\left(\epsilon_{i}\right)_{i \geq 0}$ be a stationary sequence of centered random variables with finite second moment. Let $\sigma^{2}=\operatorname{Var}\left(\epsilon_{1}\right)$ and $R$ be the correlation matrix of the vector $\left(\epsilon_{1}, \cdots, \epsilon_{n}\right)$. Consider the following regression model, defined for $i=$ $1, \cdots, n$, by

$$
Y_{i}=r\left(x_{i}\right)+\epsilon_{i}, \quad x_{i}=\frac{i}{n},
$$

where $r$ is an unknown regression function of class $\mathcal{C}^{2}$ and the $x_{i}$ 's are equally spaced fixed design. We are interested in this paper by the Priestley-Chao estimator of $r$ defined, for $x \in \mathbb{R}$, by

$$
\hat{r}(x)=\sum_{i=1}^{n} l_{i}(x) Y_{i}, \quad \text { with } \quad l_{i}(x)=\frac{1}{n h} K\left(\frac{x-x_{i}}{h}\right),
$$

where $K$ is a compactly supported even kernel with class $\mathcal{C}^{2}([-1,1])$ and $h$ is a positive bandwidth less than $1 / 2$. The above curve estimator entails the following smoothing, in the matrix form,

$$
\hat{r}=L Y \text { with } \hat{r}=\left(\hat{r}\left(x_{1}\right), \cdots, \hat{r}\left(x_{n}\right)\right)^{t}, \quad Y=\left(Y_{1}, \cdots, Y_{n}\right)^{t}
$$

and $L=\left(l_{j}\left(x_{i}\right)\right)_{1 \leq i, j \leq n}$ is known as the smoothing matrix or the hat matrix. Since the estimator $\hat{r}$ depends on some smoothing parameter $h$, we will need some procedure for choosing $h$. For this, we recall some known criteria of selecting this parameter $h$.

In order to eliminate the boundary effects of the compactly supported kernel $K$, we introduce, as was done in the literature (see for instance Gasser, T. and Muller, H.G. (1979)), a known function supported on a sub-interval of the unit interval. For this, suppose without loss of generality that $h<\epsilon$ where $\epsilon$ is a fixed positive real number less than $1 / 2$. Let $u:=u_{\epsilon}$ be a 
positive function, of class $\mathcal{C}^{1}$ and $[\epsilon, 1-\epsilon]$-compactly supported satisfying $\int_{0}^{1} u(x)\left(r^{\prime \prime}(x)\right)^{2} d x \neq 0$. Define the average squared error

$$
T_{n}(h)=\frac{1}{n} \sum_{i=1}^{n} u\left(x_{i}\right)\left(\hat{r}\left(x_{i}\right)-r\left(x_{i}\right)\right)^{2}=\frac{1}{n}\left\|U^{1 / 2}(\hat{r}-r)\right\|^{2},
$$

where $U$ is the diagonal matrix $U=\operatorname{diag}\left(u\left(x_{1}\right), \cdots, u\left(x_{n}\right)\right)$ and for any vector $v,\|v\|^{2}=v^{t} v$.

It should be pointed out that in order to overcome the boundary problem mentioned above, one may consider the local linear estimate, as done for instance by Fan, J and Gijbels, I. (1996), Francisco-Fernandez, M., Opsomer, J. and Vilar-Fernandez, J.M. (2004), Benhenni, K. and Degras, D. (2014) where plug-in asymptotic methods for selecting the smoothing parameter have been considered for some class of correlated errors. However the purpose of the current work is the study of bandwidth selection methods which are based on unbiased (or nearly unbiased) criteria for any fixed sample size. We believe that the extension of our results to local linear estimate may be carried out but it is beyond the scope of this paper and thus could be treated in a separate possible future work.

The following lemma (its proof is given in Appendix A.1 of the supplementary material) evaluates its mean, $\mathbb{E}\left(T_{n}(h)\right)$, for finite variance stationary errors $\left(\epsilon_{i}\right)_{i \in \mathbb{N}}$.

Lemma 2.1. Suppose that $\sum_{k=1}^{\infty} k\left|\operatorname{Cov}\left(\epsilon_{0}, \epsilon_{k}\right)\right|<\infty$. Define,

$$
\begin{aligned}
& D_{n}(h)=\frac{h^{4}}{4} \int_{0}^{1} u(x)\left(r^{\prime \prime}(x)\right)^{2} d x\left(\int_{-1}^{1} t^{2} K(t) d t\right)^{2} \\
& +\frac{1}{n h}\left(\int_{0}^{1} u(x) d x\right) \int_{-1}^{1} K^{2}(y) d y\left(\sigma^{2}+2 \sum_{k=1}^{\infty} \operatorname{Cov}\left(\epsilon_{0}, \epsilon_{k}\right)\right) .
\end{aligned}
$$

Then for any $n \geq 1$ and $h \in] 0, \epsilon[$,

$$
\mathbb{E}\left(T_{n}(h)\right)=D_{n}(h)+O\left(\frac{1}{n}\right)+o\left(h^{4}\right)+O\left(\frac{1}{n^{2} h^{4}}\right)+\frac{\gamma(h)}{n h},
$$

where $O$ is uniformly on $n$ and $h, \gamma(h)$ depends on $h$ (but not on $n$ ) and tends to 0 when $h$ tends to 0. 
0 ,

Let $h_{n}^{*} \in \operatorname{argmin}_{h>0} D_{n}(h)$. Clearly, since we supposed that $\int_{0}^{1} u(x)\left(r^{\prime \prime}(x)\right)^{2} d x \neq$ $h_{n}^{*}=n^{-1 / 5}\left(\frac{\left(\int_{0}^{1} u(x) d x\right) \int_{-1}^{1} K^{2}(y) d y\left(\sigma^{2}+2 \sum_{k=1}^{\infty} \operatorname{Cov}\left(\epsilon_{0}, \epsilon_{k}\right)\right)}{\int_{0}^{1} u(x)\left(r^{\prime \prime}(x)\right)^{2} d x\left(\int_{-1}^{1} t^{2} K(t) d t\right)^{2}}\right)^{1 / 5}=: c n^{-1 / 5}$.

Let, as in Hall, P., Lahiri, S. N. and Polzehl, J. (1995) and Rice, J. (1984), $H_{n}$ be a neighborhood of $h_{n}^{*}$, i.e, $H_{n}=\left[a n^{-1 / 5}, b n^{-1 / 5}\right]$ for some fixed $a<c<b$. Define also,

$$
h_{n} \in \operatorname{argmin}_{h \in H_{n}} \mathbb{E}\left(T_{n}(h)\right) \text { and } \hat{h}_{n} \in \operatorname{argmin}_{h \in H_{n}} T_{n}(h) .
$$

Of course these three "optimal" parameters $h_{n}, h_{n}^{*}$ and $\hat{h}_{n}$ depend on the unknown function $r$, since the criteria that they respectively minimise, depend themselves on the regression function $r$. Many authors agree that, among these ones, $\hat{h}_{n}$ should be the target (see Girard, D. (1998), page 316). For this reason, an important literature considered minimizers of "good" estimators of $T_{n}(h)$ and studied their asymptotic behavior.

For i.i.d. errors $\left(\epsilon_{i}\right)_{1 \leq i \leq n}$ with all finite moments, this question is solved. A reasonably good estimate of $\mathbb{E}\left(T_{n}(h)\right)$ is constructed allowing to define a criterion (of course the "goodness" can be measured up to a multiplicative positive factor or an additive constant) that selects an observable choice for $h$ : cross-validation is often used or the following simpler criterion is also used

$$
\hat{C}_{p}:=\hat{C}_{p}(h)=\frac{1}{\sum_{i=1}^{n} u\left(x_{i}\right)} \sum_{i=1}^{n} u\left(x_{i}\right)\left(Y_{i}-\hat{r}\left(x_{i}\right)\right)^{2}+2 \frac{\nu}{n} \hat{\sigma}_{h}^{2}
$$

where,

$$
\hat{\sigma}_{h}^{2}:=\frac{1}{\sum_{i=1}^{n} u\left(x_{i}\right)} \sum_{i=1}^{n} u\left(x_{i}\right)\left(Y_{i}-\hat{r}\left(x_{i}\right)\right)^{2} \text { and } \nu:=n \frac{\operatorname{tr}(U L)}{\operatorname{tr}(U)}=\frac{1}{h} K(0) .
$$

The above notation $\hat{C}_{p}$, where $\nu$ is the "local" (or more generally "weighted") "degrees of freedom", is related to the $C_{p}$-statistics introduced by Mallows, C. L. (1973) for variable selection in linear regression models. Notice that $\hat{C}_{p}(h)=\hat{\sigma}_{h}^{2} \times \Xi_{\mathrm{S}}(t(h))$ with $\Xi_{\mathrm{S}}(t):=1+2 t$ and $t(h):=\nu / n$, that means that $\hat{C}_{p}(h)$ coincides with the Shibata criterion, as named by Härdle, W., Hall, P. 
and Marron, J. S. (1988). Recall that the main result of these authors was that they showed a second-order equivalence (defined below as a footnote) of the "exact" $C_{p}$ criterion, which uses the exact $\sigma$ instead of $\hat{\sigma}_{h}$ in (2.2), and any criterion obtained by replacing in this second expression of $\hat{C}_{p}$ the penalization factor function $t \longmapsto \Xi_{\mathrm{S}}(t)$ by any function $\Xi_{\mathrm{X}}$ satisfying

$\Xi_{\mathrm{X}}(t)=1+2 t+O\left(t^{2}\right)$ with second derivative $\Xi_{\mathrm{X}}^{\prime \prime}$ bounded on a neighborhood of $0(2.3)$

a second example being the popular GCV criterion, $\hat{\sigma}_{h}^{2} /(1-t(h))^{2}$, associated with the choice $\Xi_{\mathrm{X}}(t):=\Xi_{\mathrm{GCV}}(t)=(1-t)^{-2}$. Precisely, letting $\hat{h}$ be a minimizer over $h \in H_{n}$ of the exact $C_{p}$ criterion, Härdle, W., Hall, P. and Marron, J. S. (1988) proved, in the context of i.i.d errors $\left(\epsilon_{i}\right)_{1 \leq i \leq n}$ with all finite moments, that $\hat{h}, h_{n}^{*}, \hat{h}_{n}, h_{n}$ are all equivalent in probability, that $\hat{h}-\hat{h}_{n}, h_{n}-\hat{h}_{n}$ are also close in distribution as $n$ tends to infinity, and that minimizing any such criterion $\hat{\sigma}_{h}^{2} \times \Xi_{\mathrm{X}}(t(h))$ also produces a bandwidth which is second-order equivalent to $\hat{h} .^{1}$.

The above criteria can hardly be considered as adapted to the case of general dependent errors since they take into account only the variance $\sigma^{2}$ of the errors and not their overall dependence structure. Several authors extended Mallows' criterion to some cases of stationary dependent errors. Wang, Y. (1988) and Han, Ch. and Gu, Ch. (2008), among others, generalized Mallows' criteria in (2.2) (but for other purposes than ours) to stationary dependent errors with known covariance matrix $\sigma^{2} R$ of the vector $\left(\epsilon_{1}, \cdots, \epsilon_{n}\right)^{t}$, by

$$
\mathrm{CL}(h)=n^{-1}\left\|U^{1 / 2}(I-L) Y\right\|^{2}+2 \sigma^{2} n^{-1} \operatorname{tr}(U R L),
$$

which is linked to the average squared error $T_{n}(h)$ due to the following relation,

$$
\mathrm{CL}(h)=T_{n}(h)+\delta_{2}(h)+n^{-1}\left\|U^{1 / 2}(Y-r)\right\|^{2},
$$

where

$$
\delta_{2}(h)=2 n^{-1}(Y-r)^{t} U(r-\hat{r})+2 \sigma^{2} n^{-1} \operatorname{tr}(U R L) .
$$

Let us consider, according to our purpose, $\hat{h}_{M}$ to be the minimizer of the dependent version of the Mallows criterion (2.4)

$$
\hat{h}_{M} \in \operatorname{argmin}_{h \in H_{n}} \mathrm{CL}(h) .
$$

\footnotetext{
${ }^{1}$ The second order equivalence of the $\mathrm{C}_{\mathrm{p}}$ and $\mathrm{GCV}$ selectors means that the asymptotic law of $\hat{h}-\hat{h}_{n}$ is unchanged if $\hat{h}$ is replaced by the minimizer of $\operatorname{GCV}(\mathrm{h})$
} 
Recall that we are interested in the problem of selecting the parameter $h$ when the errors form a sequence of stationary and dependent random variables. As we mentioned in the introduction, we consider through all this paper, the above regression model with stationary MDS errors (defined in Conditions (C) of Section 3 below). Since MDS is a sequence of noncorrelated and centered random variables, $R$, which represents the correlation of the errors, is nothing else but the identity matrix. Since $R=I$, it seems natural to consider again the substitution of the true $\sigma$ in (2.4) by the same estimate $\hat{\sigma}_{h}^{2}$ used above in $\hat{C}_{p}$ and to ask whether such a substitution still provides good bandwidth selectors under stationary MDS errors. Thus, we consider the following minimizers, denoted by the generic $\hat{h}_{G}$

$\hat{h}_{G} \in \operatorname{argmin}_{h \in H_{n}} G_{\mathrm{X}}(h)$ where $G_{\mathrm{X}}(h):=n^{-1}\left\|U^{1 / 2}(I-L) Y\right\|^{2} \times \Xi_{\mathrm{X}}\left(\frac{\operatorname{tr}(U L)}{\operatorname{tr}(U)}\right)(2.6)$

where $\Xi_{\mathrm{X}}$, satisfying (2.3), is associated with one of the classical GCV-type criteria.

\section{Main results and applications}

The following conditions are required to establish our main results.

Conditions (C). Assume that the errors $\left(\epsilon_{i}\right)_{i \geq 0}$ form a stationary MDS with respect to some natural filtration $\left(\mathcal{F}_{i}\right)_{i>1}$, i.e, for any $i>0, \epsilon_{i}$ is $\mathcal{F}_{i^{-}}$ measurable and $\mathbb{E}\left(\epsilon_{i} \mid \mathcal{F}_{i-1}\right)=0$. Suppose also that $\mathbb{E}\left(\epsilon_{1}^{2 p}\right)<\infty$ for some $p>8$.

Our first result states that for MDS errors, the bandwidths $h_{n}, h_{n}^{*}, \hat{h}_{n}, \hat{h}_{M}$ and $\hat{h}_{G}$ are first-order equivalent in probability (in other words, both the CL and $G_{X}$ criteria enjoy the same "asymptotic optimality" property).

Proposition 3.1. Suppose that Conditions (C) are satisfied. Then ${ }^{2}$

$$
\frac{h_{n}^{*}}{h_{n}}, \frac{\hat{h}_{n}}{h_{n}}, \frac{\hat{h}_{M}}{h_{n}}, \frac{\hat{h}_{G}}{h_{n}}
$$

all converge in probability to 1 as $n$ tends to infinity.

\footnotetext{
${ }^{2}$ Here, and for all the evoked argmin, the results apply to any points of the argmin sets
} 
Notice that Hall, P., Lahiri, S. N. and Polzehl, J. (1995) gave two theorems for two bandwidth selection methods (precisely a block-bootstrap method and the classical leave- $k$-out technique, and the mentioned theorems are respectively their Theorem 2.2 and Theorem 2.3) under a rather general dependence assumption on the error sequence, namely the Rosenblatt mixing condition (see their Section 2.2). Each of these two theorems is a first-order optimality like Proposition 3.1 above, and it could be applied, in particular, to certain stationary MDS. However we point out that these two theorems also require that all moments of the marginal law of the errors are finite. Thus the results of Hall, P., Lahiri, S. N. and Polzehl, J. (1995) cannot be applied to any $\mathrm{ARCH}$ process except the trivial one $(\alpha=0$ in the notation of Section 3.1).

Our second result gives, under a block-covariance decay condition, the rate at which $\hat{h}_{n}-\hat{h}_{M}$ and $\hat{h}_{n}-\hat{h}_{G}$ converge in distribution to a common centered normal law, and furthermore states that the martingale difference dependence does not impact this law.

Theorem 3.1. Suppose that Conditions (C) are satisfied. Moreover, suppose that there exists a positive decreasing function $\Phi$ defined on $\mathbb{R}^{+}$satisfying

$$
\sum_{s=1}^{\infty} s^{4} \Phi(s)<\infty
$$

and for any positive integer $q \leq 6,1 \leq i_{1} \leq \cdots \leq i_{k}<i_{k+1} \leq \cdots \leq i_{q} \leq n$ such that $i_{k+1}-i_{k} \geq \max _{1 \leq l \leq q-1}\left(i_{l+1}-i_{l}\right)$,

$$
\left|\operatorname{Cov}\left(\epsilon_{i_{1}} \cdots \epsilon_{i_{k}}, \epsilon_{i_{k+1}} \cdots \epsilon_{i_{q}}\right)\right| \leq \Phi\left(i_{k+1}-i_{k}\right),
$$

where $\epsilon_{i_{1}} \cdots \epsilon_{i_{k}}$ denotes the product $\prod_{\ell=1}^{k} \epsilon_{i_{\ell}}$ (and likewise for $\epsilon_{i_{k+1}} \cdots \epsilon_{i_{q}}$ ). Then both

$$
n^{3 / 10}\left(\hat{h}_{M}-\hat{h}_{n}\right) \text { and } n^{3 / 10}\left(\hat{h}_{G}-\hat{h}_{n}\right)
$$

converge in distribution to a centered normal law with variance $\Sigma^{2}$ given by

$\Sigma^{2}=\frac{4 \sigma^{6 / 5}}{5^{2} A^{8 / 5} B^{2 / 5}}\left(\left(\int t^{2} K(t) d t\right)^{2} \int_{0}^{1} u^{2}(x) r^{\prime \prime 2}(x) d x+\frac{2 A}{B} \int_{0}^{1} u^{2}(x) d x \int(K-G)^{2}(t) d t\right)$,

where $\sigma^{2}=\mathbb{E}\left(\epsilon_{1}^{2}\right), G$ is the function defined for any $x \in \mathbb{R}$ by $G(x)=$ $-x K^{\prime}(x)$ and

$$
A=\int_{0}^{1} u(x) r^{\prime \prime 2}(x) d x\left(\int t^{2} K(t) d t\right)^{2}, B=\int_{0}^{1} u(x) d x \int K^{2}(t) d t .
$$


Remark 3.1. The control of the covariance quantity $\left|\operatorname{Cov}\left(\epsilon_{i_{1}} \cdots \epsilon_{i_{k}}, \epsilon_{i_{k+1}} \cdots \epsilon_{i_{q}}\right)\right|$ appearing in (3.1) is well known in the literature. It was used, for instance in Doukhan, P. and Louhichi, S. (1999), in order to obtain MarcinkiewiczZygmund type moments inequalities of an even order of the partial sum $\sum_{i=1}^{n} \epsilon_{i}$. If the sequence $\left(\epsilon_{n}\right)_{n}$ is strongly mixing with mixing coefficients $\left(\alpha_{s}\right)_{s \in \mathbb{N}}$, then it is proved by Rio, E. (1993), see also Lemma 9 in Doukhan, P. and Louhichi, S. (1999)) that, for $1 \leq i_{1} \leq \cdots \leq i_{k}<i_{k+1} \leq \cdots \leq i_{q} \leq n$ such that $s:=i_{k+1}-i_{k} \geq \max _{1 \leq l \leq q-1}\left(i_{l+1}-i_{l}\right)$,

$$
\left|\operatorname{Cov}\left(\epsilon_{i_{1}} \cdots \epsilon_{i_{k}}, \epsilon_{i_{k+1}} \cdots \epsilon_{i_{q}}\right)\right| \leq 4 \int_{0}^{\alpha_{s}} Q^{q}(u) d u,
$$

where $Q$ is the quantile function of $\left|\epsilon_{1}\right|$, i.e. the inverse of the tail function $t \longmapsto \mathbb{P}\left(\left|\epsilon_{1}\right|>t\right)$.

\subsection{Application to $\mathrm{ARCH}(1)$ processes}

We consider the regression model defined in (2.1) with an ARCH(1) error process $\left(\epsilon_{n}\right)_{n \geq 1}$ defined, for $n \geq 1$, by the following stochastic difference equation,

$$
\epsilon_{n}=\eta_{n} \sqrt{\sigma^{2}(1-\alpha)+\alpha \epsilon_{n-1}^{2}}, 0 \leq \alpha<1, \sigma^{2}>0
$$

where $\left(\eta_{n}\right)_{n \geq 1}$ is an i.i.d. centered sequence distributed as a standard normal law and such that $\eta_{n}$ is independent of $\left(\epsilon_{1}, \cdots, \epsilon_{n-1}\right)$.

Proposition 3.2. Let $\left(\epsilon_{n}\right)_{n \geq 1}$ be a strictly stationary ARCH(1) process satisfying (3.2) with $\alpha$ such that $\alpha^{8} \prod_{i=1}^{8}(2 i-1)<1$ (this is equivalent to $\alpha<2025027^{-1 / 8} \approx 0.162796$ ). Then the conclusions of Proposition 3.1 and Theorem 3.1 hold.

Proof of Proposition 3.2. We first recall the following well known properties in the literature (see for instance Engle, R. F. (1982), Lindner, A.M. (2009) and the references therein).

Lemma 3.1. Consider the process $\left(\epsilon_{n}\right)_{n}$ as defined in (3.2). Then

1. $\left(\epsilon_{n}\right)_{n}$ is a geometric ergodic homogeneous Markov chain with a unique stationary distribution $\pi$. The stationary distribution $\pi$ is continuous and symmetric. 
2. $\left(\epsilon_{n}\right)_{n}$ is strongly mixing with mixing coefficients $\left(\alpha_{l}\right)_{l>0}$

$$
\alpha_{l}:=\sup _{A \in \sigma\left(\epsilon_{s}, s \leq 0\right), B \in \sigma\left(\epsilon_{s}, s \geq l\right)}\left|\operatorname{Cov}\left(\mathbb{I}_{A}, \mathbb{I}_{B}\right)\right|=O\left(\rho^{l}\right),
$$

for some $\rho \in] 0,1\left[\right.$. Here $\sigma\left(\epsilon_{s} ; s \leq 0\right)$ and $\sigma\left(\epsilon_{s} ; s \geq l\right)$ denote, respectively the sigma-fields generated by $\epsilon_{s}$, for $s \leq 0$ and $\epsilon_{s}$, for $s \geq l$.

3. $\mathbb{E}\left(\epsilon_{1}^{2 r}\right)<\infty$, for $r \in \mathbb{N} \backslash\{0\}$, if and only if $\alpha^{r} \prod_{i=1}^{r}(2 i-1)<1$.

4. $\mathbb{P}\left(\left|\epsilon_{1}\right|>x\right) \sim c x^{-\kappa}$ as $x$ tends to infinity (in all this paper the notation $a(x) \sim b(x)$ means that $\left.\lim _{x \rightarrow \infty} \frac{a(x)}{b(x)}=1\right)$, for some $c>0$ and $\kappa$ is given as the unique positive solution to $\alpha^{\kappa / 2} \mathbb{E}\left(\left|\eta_{1}\right|^{\kappa}\right)=1$.

Letting $\mathcal{F}_{i}=\sigma\left(\eta_{1}, \cdots, \eta_{i}\right)$, then $\epsilon_{i}$ is $\mathcal{F}_{i}$-measurable and

$$
\mathbb{E}\left(\epsilon_{i} \mid \mathcal{F}_{i-1}\right)=\sqrt{\sigma^{2}(1-\alpha)+\alpha \epsilon_{i-1}^{2}} \mathbb{E}\left(\eta_{i} \mid \mathcal{F}_{i-1}\right)=0
$$

The sequence $\left(\epsilon_{n}\right)_{n}$ is then a martingale-difference. Moreover, since it is strongly mixing with $\alpha_{s} \leq C \rho^{s}$, we get, from Remark 3.1, the bound (3.1), for any given $q$, by using

$$
\Phi(s):=4 \int_{0}^{\alpha_{s}} Q^{q}(u) d u .
$$

Our task now is to prove that $\sum_{s=1}^{\infty} s^{4} \Phi(s)<\infty$. We deduce from $\mathbb{P}\left(\left|\epsilon_{1}\right|>\right.$ $x) \sim c x^{-\kappa}$ as $x$ tends to infinity that $Q(u)=O\left(u^{-1 / \kappa}\right)$, ( $q$ satisfies necessarily $q<\kappa$, since $\left.\mathbb{E}\left(\left|\epsilon_{1}\right|^{q}\right)<\infty\right)$ and

$$
\int_{0}^{\alpha_{s}} Q^{q}(u) d u \leq \int_{0}^{C \rho^{s}} Q^{q}(u) d u=O\left(\rho^{s(1-q / \kappa)}\right)
$$

Consequently, for some positive constant $C$,

$\sum_{s=1}^{\infty} s^{4} \Phi(s) \leq 4 \sum_{s=1}^{\infty} s^{4} \int_{0}^{C \rho^{s}} Q^{q}(u) d u \leq C \sum_{s=1}^{\infty} s^{4} \rho^{s(1-q / \kappa)}<\infty$, since $\left.\rho \in\right] 0,1[$. 


\subsection{A Monte-carlo simulation study for a "trend plus $\operatorname{ARCH}(1)$ process"}

We report here on rather extensive experiments with $\mathrm{ARCH}(1)$ noise and a single example of regression function (called the "deterministic trend" here) taken from Welsh, A.H., Lin, X. and Carroll, R.J. (2002), and we focus on the questions of how accurate is the approximation provided by Theorem 3.1, and whether the restriction $p>8$ in Conditions (C) that we have required could be relaxed. These questions were also studied, in the first arXiv version of this article, for another trend, namely the well known "bell shaped" example much studied since Rice, J. (1984), which is a lot smoother trend than the one used here. And for the sake of place we only do this experimental study for the Mallows criterion (the possible GCV-like criteria, satisfying (2.3), being rather numerous, cf. Härdle, W., Hall, P. and Marron, J. S. (1988)). We choose a noise level for which the noise-to-signal ratio is "moderate", precisely $0.32^{2}$. So, the chosen trend function is

$$
r(x)=c_{0}+c_{1}\left(\sin (8 x-4)+2 \exp \left(-256(x-0.5)^{2}\right)\right)
$$

where we add the constants $c_{0}, c_{1}$ to the definition by Welsh, A.H., Lin, X. and Carroll, R.J. (2002) only so that the range of $r(x)$ is exactly [0, 1] when $x \in[0,1])$ : a plot of $r$ is inserted in the left panel of Figure 1. Each data set is thus the sum of this trend $r$ evaluated at $x_{i}=i / n, i=1, \cdots, n$, plus an $\operatorname{ARCH}(1)$ sequence with a "persistence" parameter $\alpha$ as defined in the above Subsection 3.1. We consider 6 settings for the $\mathrm{ARCH}(1)$ noise, precisely

$\alpha \in\{0.01,0.162,0.577,0.75,0.9,0.98\}$, with a common value $\sigma=0.32$.

The first value of $\alpha=0.01$ corresponds nearly to i.i.d. normal observation noises (this setting will be referred to as the "quasi-iid-normal" case) and the last one generates noise sequences for which a strong serial correlation is always present when the sequence is squared. Recall that the moment of order 16 no longer exists as soon as $\alpha$ is slightly above 0.162 , but the moment of order 4 still exists for $\alpha<\sqrt{1 / 3} \approx 0.57735$. 

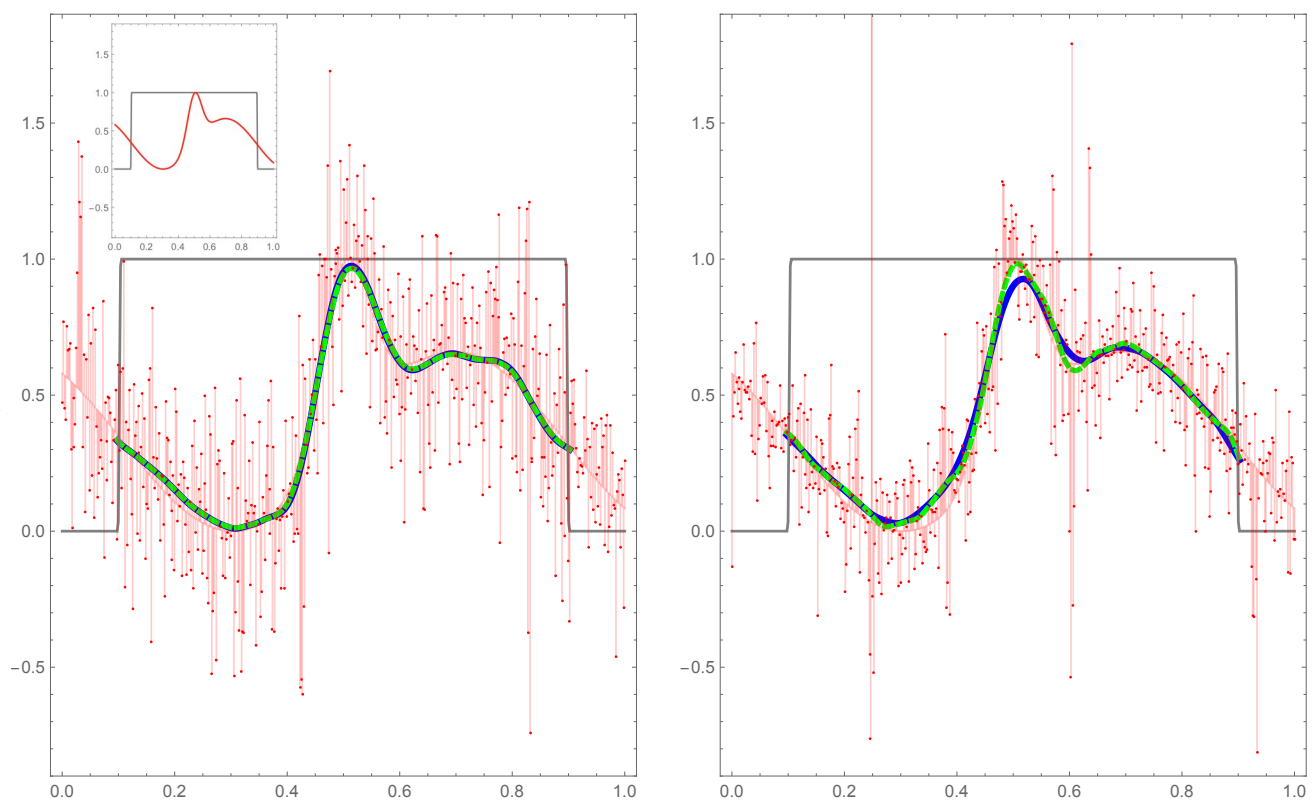

Figure $1: n=2^{9}$. Each of these 2 panels displays one data set $Y$. The underlying trend $r$ is displayed (in red) in the upper left inset. The function $u$ (in gray) excludes about $20 \%$ of the points. The 2 panels only differ by $\alpha=0.577$ (left) and $\alpha=0.9$ (right). For each $Y$ the CL choice and the "weighted $L_{2}$-optimal" $T_{n}$ choice are plotted in blue and dashed green respectively.

The kernel function used here is the classical biweight $K(x)=\frac{15}{16}(1-$ $\left.x^{2}\right)^{2} 1_{[-1,1]}(x)$. As is well known, its precise specification, among possible positive "bell shaped" kernels, has a weak impact on the behavior of bandwidth selection techniques. As weight function $u$, we used a slightly smoothed version of $\mathbb{1}_{[0.1,0.9]}$. Since its precise specification also has a weak impact, we only give a plot of the used $u$ in Figure 1. Notice that for the considered data sizes $n$ here, it turns out that it is sufficient to consider only bandwidths that are lower than 0.1 , as candidate bandwidths. Then, since the bandwidth $h$ is one-half the support length of $\frac{1}{h} K(\dot{h})$, it can be checked that computing the sub-vector of the Priestley-Chao estimator $L Y$ whose components are restricted to the $x_{i}$ 's in $[0.1,0.9]$, can always be done by discrete Fourier transforms. This remark makes affordable the following simulation study even for quite large $n$.

The data sets size $n$ was chosen in $\left\{2^{9}, 2^{12}, 2^{15}\right\}=\{512,4096,32768\}$. We generated 1000 replicated data sets for each of these $3 \times 6$ settings. For each data set, the minimizer of $T_{n}(h)$ and the one of CL $(h)$ were numerically 
computed by a simple grid-search over the domain $[0.025,1] \times 10^{-1}$ (notice the "no smoothing $h$ " is $n^{-1} K(0) \approx 0.0018$ for $n=512$ and when $h$ comes close to $1 / 2$ one averages over the entire sample, see Härdle, W., Hall, P. and Marron, J. S. (1988)), the grid-step being chosen fine enough so that the "granularity" in the 2000 computed $h$ 's has a very weak impact on the conclusions.
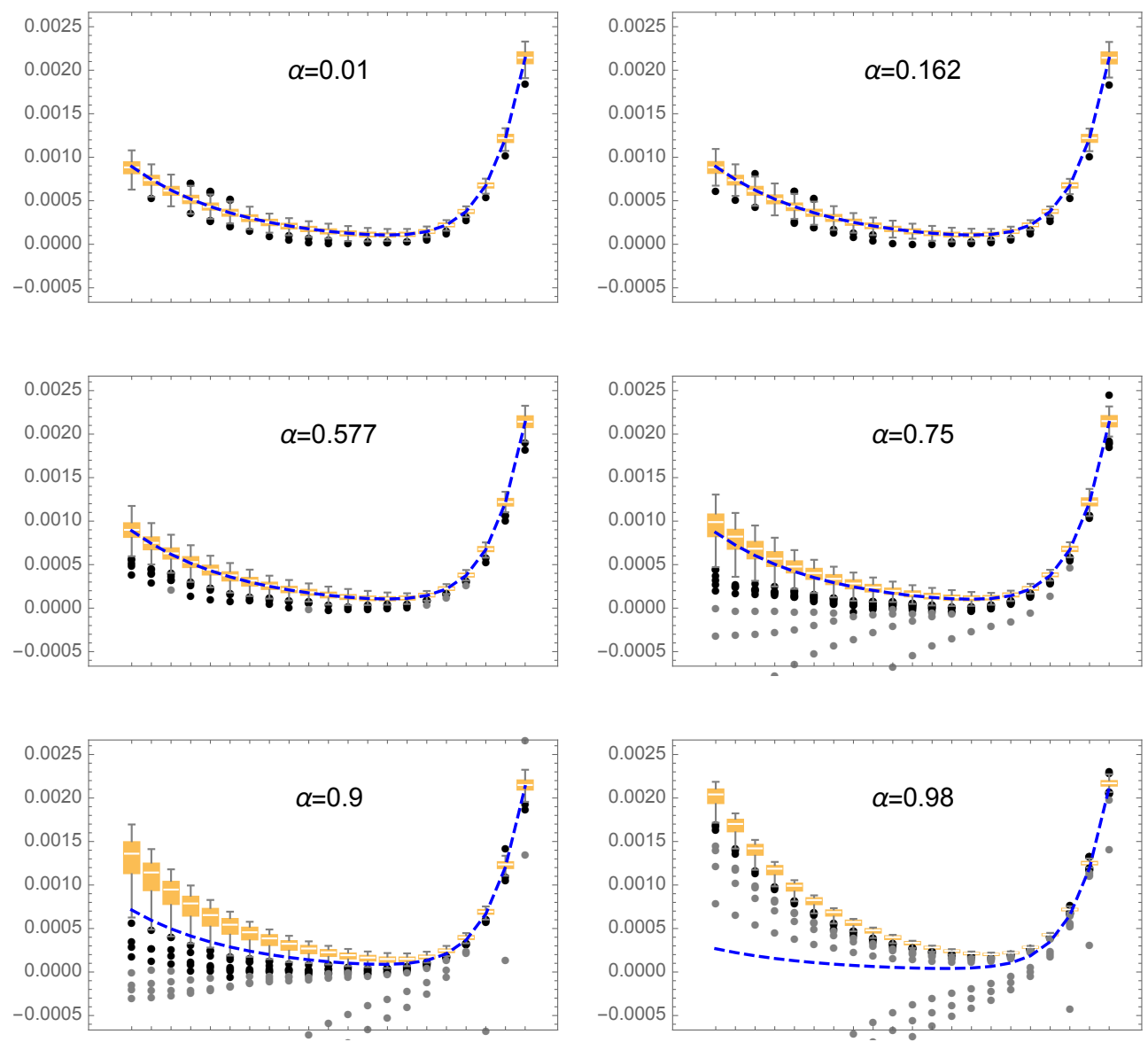

Figure $2: n=2^{15}$. These 6 panels only differ by $\alpha$ varying in $\{0.01,0.162,0.577,0.75,0.9,0.98\}$. In each panel, the dashed blue curve is "empirical MASE", precisely the average (over the 1000 replicates) of the $T_{n}(h)$ curves. Each of the 21 boxplots (located at 21 discrete values for $h$ equispaced over $[0.025,1] \times 10^{-1}$ ) are built from the 1000 replicates of $\mathrm{CL}(h)-n^{-1}\left\|U^{1 / 2}(Y-r)\right\|^{2}$. 
The "a.o." property. First, let us analyze the asymptotic optimality (a.o.) result. As is well known, a result like Proposition 3.1 generally stems from a uniform relative accuracy result which states that $\mathrm{CL}(h)-n^{-1}\left\|U^{1 / 2}(Y-r)\right\|^{2}$ uniformly approximates $T_{n}(h)$ (or its expectation $\operatorname{MASE}(h)$ ) with a small (in probability and in sup norm over the domain of candidate $h$ 's) error, "small" being defined relatively to $\operatorname{MASE}(h)$.

We resume in Figure 2 that a uniform relative accuracy is well observed and, above all, this accuracy in the case $\alpha=0.162$ is of the same order as the accuracy observed in the quasi-iid-normal case $(\alpha=0.01)$. Furthermore, an interesting observation is that this accuracy is not deteriorated when $\alpha=0.577$. However there is clearly a deterioration for larger $\alpha$, especially for $\alpha=0.9$ or 0.98 where in addition to the increased variability, a large bias is observed. Figure 1 exhibits such a bias toward oversmoothing for $\alpha=0.9$.

It can be thus conjectured that, at least for $\mathrm{ARCH}(1)$ processes, the restriction $p>8$ of our Conditions (C) might be weakened to $p>2$. However, the poor behavior of CL (even with quite large $n$ ) in cases $\alpha=0.75,0.9$ or 0.98 , leads us to conjecture that $p>2$ should be considered as a necessary condition for the a.o. of CL or GCV under general stationary MDS observation errors.

Asymptotic normal distribution. Now, let us look at the usefulness of the asymptotic normal approximation stated in Theorem 3.1. By inspecting Figure 3, we clearly see, in the left-bottom panel, that this approximation fits very well for $n=2^{15}$ and $\alpha=0.577$. 

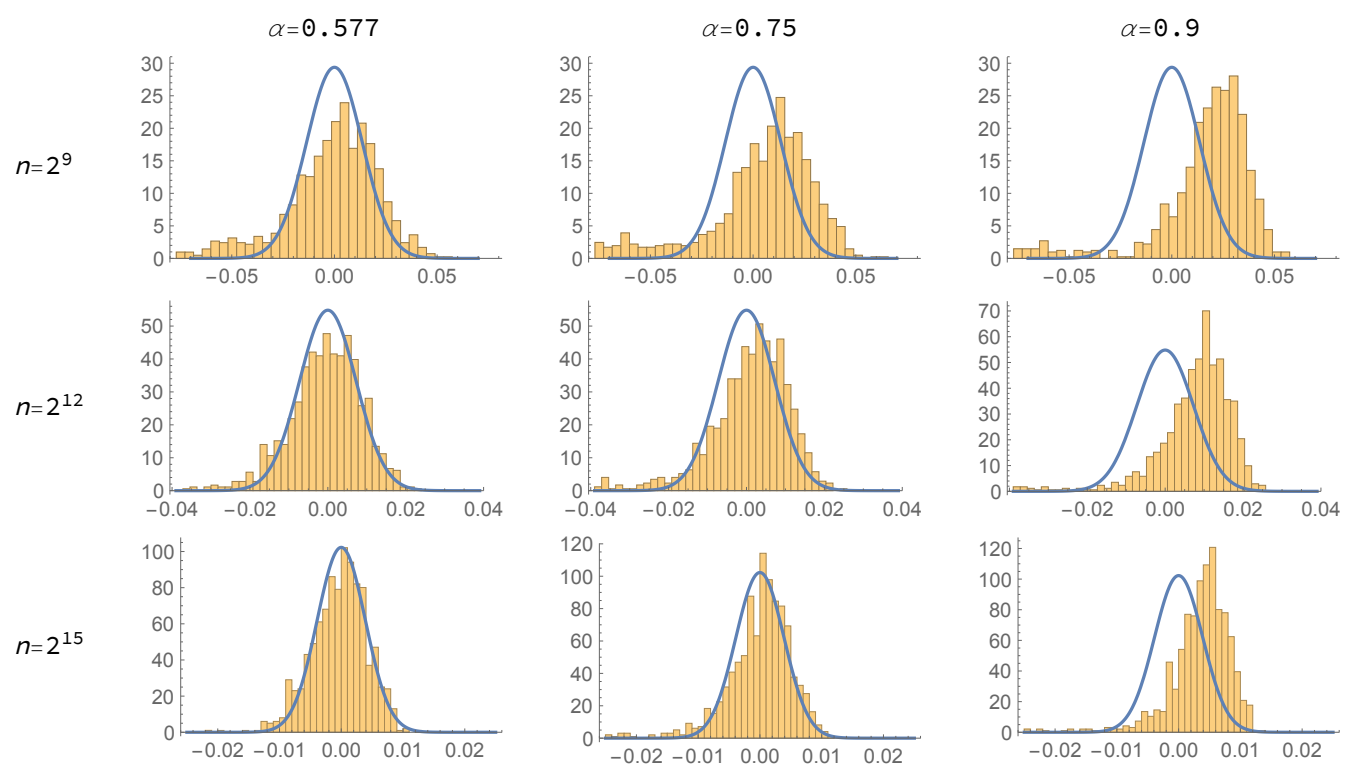

Figure $3: n=512$ (top), 4096 (middle) and 32768 (bottom). These 9 panels only differ by $n$ and by $\alpha$ varying in $\{0.577,0.75,0.9\}$. In each panel, the displayed histogram is that of the 1000 replicates of $\hat{h}_{M}-\hat{h}_{n}$; the histograms are normalized so that their integrals are equal to 1 . The superposed blue curve is the normal distribution of $\hat{h}_{M}-\hat{h}_{n}$ predicted by the asymptotic theory. Notice that, as expected, the range of the abscissae ( $h$-differences) decreases by moving from $n=2^{9}$ to $n=2^{15}$.

We have also made such a comparison for $\alpha=0.01$ and $\alpha=0.162$ (not shown in Figure 3), and, as expected by Theorem 3.1 and Section 3.1, the fit is also very good. For settings with the much smaller $n=512$, the fit is still rather good for $\alpha=0.577$, but this is no longer true for $\alpha=0.75$ or $\alpha=0.9$ (and the fit is even worse for $\alpha=0.98$, not shown in Figure 3). For $n=2^{12}$ we see that the accuracy of the fit, when $\alpha=0.577$, is almost as good as in the case $n=2^{15}$. Similar conclusions were obtained for the "bell shaped" trend mentioned above. It is good news that the asymptotic approximation given by Theorem 3.1 is thus useful also with $\alpha=0.577$, since this gives support to the conjecture that Theorem 3.1 could be extended to an ARCH process under the only existence of the fourth moment of the marginal law. 


\section{Proofs}

\subsection{Main lemmas for the proof of Proposition 3.1}

The following two lemmas are very useful for the proof of Proposition 3.1. Their proofs use the tools (stated in Appendix $\mathbf{C}$ ), that control the supremum of the higher moments of sums of weighted MDS or quadratic form of weighted MDS, since the evoked quantities $\delta_{2}(h), T_{n}(h)$ and their derivatives are expressed in terms of sums of weighted MDS or quadratic form of weighted MDS.

We denote by, $\|\cdot\|_{p}$ the $p$-norm, i.e, for a random variable $X,\|X\|_{p}=$ $\left(\mathbb{E}\left(|X|^{p}\right)\right)^{1 / p}$ and we recall that $\delta_{2}(h)$ is defined as in $(2.5)$ and $H_{n}=\left[a n^{-1 / 5}, b n^{-1 / 5}\right]$ for some fixed $a<c<b$.

Lemma 4.1. It holds, for $p>8$,

$$
\begin{aligned}
& \lim _{n \rightarrow \infty}\left\|\sup _{h \in H_{n}} n h\left|\delta_{2}(h)\right|\right\|_{p}=0 \\
& \lim _{n \rightarrow \infty}\left\|\sup _{h \in H_{n}} n h^{2}\left|\delta_{2}^{\prime}(h)\right|\right\|_{p}=0 \\
& \lim _{n \rightarrow \infty}\left\|\sup _{h \in H_{n}} n h^{3}\left|\delta_{2}^{\prime \prime}(h)\right|\right\|_{p}=0 \\
& \lim _{M \rightarrow \infty} \limsup _{n \rightarrow \infty} \mathbb{P}\left(\sup _{h \in A_{\epsilon}} \sqrt{n}\left|\delta_{2}^{\prime \prime}(h)\right| \geq M\right)=0
\end{aligned}
$$

where for fixed $\epsilon>0, A_{\epsilon}$ is a subset of $H_{n}$ defined by,

$$
A_{\epsilon}=\left\{h \in H_{n},\left|\frac{h}{h_{n}}-1\right| \leq \epsilon\right\} .
$$

Lemma 4.2. It holds, for $p>8$,

$$
\begin{aligned}
& \lim _{n \rightarrow \infty}\left\|\sup _{h \in H_{n}} n h\left|T_{n}(h)-\mathbb{E}\left(T_{n}(h)\right)\right|\right\|_{p}=0, \\
& \lim _{n \rightarrow \infty}\left\|\sup _{h \in H_{n}} n h^{2}\left|T_{n}^{\prime}(h)-\mathbb{E}\left(T_{n}^{\prime}(h)\right)\right|\right\|_{p}=0, \\
& \lim _{n \rightarrow \infty}\left\|\sup _{h \in H_{n}} n h^{3}\left|T_{n}^{\prime \prime}(h)-\mathbb{E}\left(T_{n}^{\prime \prime}(h)\right)\right|\right\|_{p}=0 .
\end{aligned}
$$




\subsubsection{Proof of Lemma 4.1}

We have the following decomposition

$$
\begin{aligned}
& \delta_{2}(h)=\left(2 n^{-1}(Y-r)^{\prime} U(r-\hat{r})+2 \sigma^{2} n^{-1} \operatorname{tr}(U R L)\right) \\
& =2 \sum_{i=1}^{n} A_{i}(h) u\left(x_{i}\right) \epsilon_{i}+2 \sum_{i=1}^{n} \sum_{j=1}^{i-1}\left(u\left(x_{i}\right)+u\left(x_{j}\right)\right) B_{i, j}(h) \epsilon_{i} \epsilon_{j}+2 \sum_{i=1}^{n} u\left(x_{i}\right) B_{i, i}(h)\left(\epsilon_{i}^{2}-\mathbb{E}\left(\epsilon_{i}^{2}\right)\right),
\end{aligned}
$$

with,

$$
\begin{aligned}
& A_{i}(h)=\frac{1}{n}\left(r\left(x_{i}\right)-\mathbb{E}\left(\hat{r}\left(x_{i}\right)\right)\right)=-\frac{1}{n} B\left(x_{i}, h\right) \\
& B_{i, j}(h)=-n^{-1} \frac{1}{n h} K\left(\frac{x_{i}-x_{j}}{h}\right), \quad B_{i, i}(h)=-n^{-1} \frac{1}{n h} K(0) .
\end{aligned}
$$

Proof of (4.1). We have, for any $h, h^{\prime} \in H_{n}$, (using the same calculations yielding to (A.11) of the supplementary material),

$$
\begin{aligned}
& u\left(x_{i}\right) \sup _{h \in H_{n}} n h\left|A_{i}(h)\right|=O\left(n^{-3 / 5}\right), \\
& u\left(x_{i}\right)\left|n h A_{i}(h)-n h^{\prime} A_{i}\left(h^{\prime}\right)\right| \\
& \leq h u\left(x_{i}\right)\left|B\left(x_{i}, h\right)-B\left(x_{i}, h^{\prime}\right)\right|+u\left(x_{i}\right) B\left(x_{i}, h^{\prime}\right)\left|h-h^{\prime}\right| \leq c s t n^{-2 / 5}\left|h-h^{\prime}\right|, \\
& \\
& n h\left|B_{i, j}(h)\right| \leq \operatorname{cst}^{-1} \mathbb{1}_{|i-j| \leq n h}, \\
& \quad\left|n h B_{i, j}(h)-n h^{\prime} B_{i, j}\left(h^{\prime}\right)\right| \leq c s t n^{-4 / 5}\left|h-h^{\prime}\right| \mathbb{I}_{|i-j| \leq n \max \left(h, h^{\prime}\right)},
\end{aligned}
$$

and $n h B_{i, i}(h)=\frac{K(0)}{n}$. Using Lemmas C.1, C.3 of the supplementary material and the fact that

$$
\frac{1}{n} \sum_{i=1}^{n} u\left(x_{i}\right)\left(\epsilon_{i}^{2}-\mathbb{E}\left(\epsilon_{i}^{2}\right)\right) \rightarrow 0, \text { in probability as } n \rightarrow \infty,
$$

we get

$$
\lim _{n \rightarrow \infty} \sup _{h \in H_{n}} n h\left|\delta_{2}(h)\right|=0 \text {, in probability as } n \rightarrow \infty \text {. }
$$

Proofs of (4.2) and (4.3). We only discuss the proof (4.3) since that of (4.2) is similar. For any $h \in] 0, \epsilon[$ and any $n \geq 1$,

$\delta_{2}^{\prime \prime}(h)=2 \sum_{i=1}^{n} c_{i}(h) u\left(x_{i}\right) \epsilon_{i}+2 \sum_{i=1}^{n} \sum_{j=1}^{i-1}\left(u\left(x_{i}\right)+u\left(x_{j}\right)\right) c_{i, j}(h) \epsilon_{i} \epsilon_{j}+2 \sum_{i=1}^{n} c_{i, i}(h) u\left(x_{i}\right)\left(\epsilon_{i}^{2}-\mathbb{E}\left(\epsilon_{i}^{2}\right)\right)$, 
where, letting $K_{1}=K-G$ and $G_{1}(u)=-u K_{1}^{\prime}(u)$,

$$
\begin{aligned}
& c_{i}(h)=-\frac{1}{n} \frac{\partial^{2}}{\partial h^{2}} \mathbb{E}\left(\hat{r}\left(x_{i}\right)\right)=-\frac{1}{n} \frac{\partial^{2}}{\partial h^{2}} B\left(x_{i}, h\right), \\
& c_{i, j}(h)=-\frac{2}{n^{2} h^{3}} K_{1}\left(\frac{x_{i}-x_{j}}{h}\right)+\frac{1}{n^{2} h^{3}} G_{1}\left(\frac{x_{i}-x_{j}}{h}\right), \text { and } c_{i, i}(h)=\frac{-2}{n^{2} h^{3}} K(0) .
\end{aligned}
$$

Now,

$$
n h^{3} \sum_{i=1}^{n} c_{i, i}(h) u\left(x_{i}\right)\left(\epsilon_{i}^{2}-\mathbb{E}\left(\epsilon_{i}^{2}\right)\right)=\operatorname{cst} \frac{1}{n} \sum_{i=1}^{n} u\left(x_{i}\right)\left(\epsilon_{i}^{2}-\mathbb{E}\left(\epsilon_{i}^{2}\right)\right)
$$

which converges in probability to 0 by an analogous to Lemma 4.4 .

We also have, for any $h, h^{\prime} \in H_{n}$, (see Lemma A.3 of the supplementary material) the following bounds,

$$
\begin{aligned}
& u\left(x_{i}\right)\left|n h^{3} c_{i}(h)\right| \leq \operatorname{cst} n^{-3 / 5}, \\
& u\left(x_{i}\right)\left|n h^{3} c_{i}(h)-n h^{\prime 3} c_{i, n}\left(h^{\prime}\right)\right| \leq \operatorname{cstn}^{-2 / 5}\left|h-h^{\prime}\right|, \\
& u\left(x_{i}\right)\left|n h^{3} c_{i, j}(h)\right| \leq \operatorname{cst} \frac{1}{n} \mathbb{I}_{|i-j| \leq n h}, \\
& u\left(x_{i}\right)\left|n h^{3} c_{i, j}(h)-n h^{\prime 3} c_{i, j}\left(h^{\prime}\right)\right| \leq \operatorname{cstn}^{-4 / 5}\left|h-h^{\prime}\right| \mathbb{I}_{|i-j| \leq n \max \left(h, h^{\prime}\right)} .
\end{aligned}
$$

All the requirements of Lemmas C.1 and C.3 of the supplementary material are satisfied. We deduce that,

$$
\lim _{n \rightarrow \infty} \sup _{h \in H_{n}} n h^{3}\left|\delta_{2}^{\prime \prime}(h)\right|=0, \text { in probability. }
$$

Proof of (4.4). We have,

$$
\begin{aligned}
& \sqrt{n} \delta_{2}^{\prime \prime}(h)=2 \sum_{i=1}^{n} e_{i}(h) u\left(x_{i}\right) \epsilon_{i}+2 \sum_{i=1}^{n} \sum_{j=1}^{i-1}\left(u\left(x_{i}\right)+u\left(x_{j}\right)\right) e_{i, j}(h) \epsilon_{i} \epsilon_{j} \\
& +2 \sum_{i=1}^{n} e_{i, i}(h) u\left(x_{i}\right)\left(\epsilon_{i}^{2}-\mathbb{E}\left(\epsilon_{i}^{2}\right)\right),
\end{aligned}
$$

where,

$$
\begin{aligned}
& e_{i}(h)=-\frac{1}{\sqrt{n}} \frac{\partial^{2}}{\partial h^{2}} \mathbb{E}\left(\hat{r}\left(x_{i}\right)\right)=-\frac{1}{\sqrt{n}} \frac{\partial^{2}}{\partial h^{2}} B\left(x_{i}, h\right), \\
& e_{i, j}(h)=-\frac{2}{n \sqrt{n} h^{3}} K_{1}\left(\frac{x_{i}-x_{j}}{h}\right)+\frac{1}{n \sqrt{n} h^{3}} G_{1}\left(\frac{x_{i}-x_{j}}{h}\right), \\
& e_{i, i}(h)=\frac{-2}{n \sqrt{n} h^{3}} K(0) .
\end{aligned}
$$


We have, since $\sum_{i=1}^{\infty}\left|\operatorname{Cov}\left(\epsilon_{1}^{2}, \epsilon_{i}^{2}\right)\right|<\infty$,

$$
\begin{aligned}
& \sup _{h \in H_{n}}\left|\sum_{i=1}^{n} e_{i, i}(h) u\left(x_{i}\right)\left(\epsilon_{i}^{2}-\mathbb{E}\left(\epsilon_{i}^{2}\right)\right)\right| \leq \frac{c s t}{n^{9 / 10}}\left|\sum_{i=1}^{n} u\left(x_{i}\right)\left(\epsilon_{i}^{2}-\mathbb{E}\left(\epsilon_{i}^{2}\right)\right)\right| \\
& \left\|\sup _{h \in H_{n}}\left|\sum_{i=1}^{n} e_{i, i}(h) u\left(x_{i}\right)\left(\epsilon_{i}^{2}-\mathbb{E}\left(\epsilon_{i}^{2}\right)\right)\right|\right\|_{2}^{2} \leq \frac{c s t}{n^{18 / 10}}\left\|\sum_{i=1}^{n} u\left(x_{i}\right)\left(\epsilon_{i}^{2}-\mathbb{E}\left(\epsilon_{i}^{2}\right)\right)\right\|_{2}^{2} \\
& \leq \operatorname{cst} \frac{n}{n^{18 / 10}} .
\end{aligned}
$$

Hence,

$$
\lim _{n \rightarrow \infty}\left\|\sup _{h \in H_{n}}\left|\sum_{i=1}^{n} e_{i, i}(h) u\left(x_{i}\right)\left(\epsilon_{i}^{2}-\mathbb{E}\left(\epsilon_{i}^{2}\right)\right)\right|\right\|_{2}=0 .
$$

Let, for $h \in H_{n}, h^{\prime} \in H_{n}, \tilde{e}_{i}(h)=e_{i}(h)-e_{i}\left(h_{n}\right)$, then

$$
\begin{aligned}
& u\left(x_{i}\right)\left|\tilde{e}_{i}(h)\right| \leq \operatorname{cst} \frac{1}{\sqrt{n}}\left|h-h_{n}\right| \leq \operatorname{cst} n^{-7 / 10}, \\
& u\left(x_{i}\right)\left|\tilde{e}_{i}(h)-\tilde{e}_{i}\left(h^{\prime}\right)\right| \leq \operatorname{cst} \frac{1}{\sqrt{n}}\left|h-h^{\prime}\right|
\end{aligned}
$$

Hence,

$$
\sup _{h \in H_{n}}\left|\sum_{i=1}^{n} e_{i}(h) u\left(x_{i}\right) \epsilon_{i}\right| \leq \sup _{h \in H_{n}}\left|\sum_{i=1}^{n}\left(e_{i}(h)-e_{i}\left(h_{n}\right)\right) u\left(x_{i}\right) \epsilon_{i}\right|+\left|\sum_{i=1}^{n} e_{i}\left(h_{n}\right) u\left(x_{i}\right) \epsilon_{i}\right| .
$$

It follows that

$$
\begin{aligned}
& \left\|\sup _{h \in H_{n}}\left|\sum_{i=1}^{n} e_{i}(h) u\left(x_{i}\right) \epsilon_{i}\right|\right\|_{p} \\
& \leq\left\|\sup _{h \in H_{n}}\left|\sum_{i=1}^{n}\left(e_{i}(h)-e_{i}\left(h_{n}\right)\right) u\left(x_{i}\right) \epsilon_{i}\right|\right\|_{p}+\left\|\sum_{i=1}^{n} e_{i}\left(h_{n}\right) u\left(x_{i}\right) \epsilon_{i}\right\|_{p} .
\end{aligned}
$$

Applying Lemmas C.1 and Corollary C.1 of Appendix $\mathbf{C}$ of the supplementary material, we deduce that,

$$
\limsup _{n \rightarrow \infty}\left\|\sup _{h \in H_{n}} \mid \sum_{i=1}^{n} e_{i}(h) u\left(x_{i}\right) \epsilon_{i}\right\| \|_{p}<\infty
$$


Let, for $h \in A_{\epsilon}=\left\{h \in H_{n},\left|\frac{h}{h_{n}}-1\right| \leq \epsilon\right\}, H_{i, j}(h)=\frac{1}{h^{3}}\left(G_{1}-2 K_{1}\right)\left(\frac{x_{i}-x_{j}}{h}\right)$

$$
\tilde{e}_{i, j}(h)=e_{i, j}(h)-e_{i, j}\left(h_{n}\right)=\frac{1}{n^{3 / 2}}\left(H_{i, j}(h)-H_{i, j}\left(h_{n}\right)\right) .
$$

Since, $\left|\frac{\partial H_{i, j}}{\partial h}(h)\right| \leq \operatorname{cst}^{-4}$, then for any $h, h^{\prime} \in H_{n}$

$$
\left|H_{i, j}(h)-H_{i, j}\left(h^{\prime}\right)\right| \leq n^{4 / 5}\left|h-h^{\prime}\right| \mathbb{1}_{|i-j| \leq n \max \left(h, h^{\prime}\right)}
$$

and, for any $h \in A_{\epsilon}$,

$$
\begin{aligned}
& \left|\tilde{e}_{i, j}(h)\right| \leq \operatorname{cst} \frac{\epsilon}{n^{9 / 10}} \mid \mathbb{I}_{|i-j| \leq n \max \left(h, h^{\prime}\right)}, \\
& \left|\tilde{e}_{i, j}(h)-\tilde{e}_{i, j}\left(h^{\prime}\right)\right| \leq \operatorname{cst} \frac{1}{n^{7 / 10}}\left|h-h^{\prime}\right| \mathbb{1}_{|i-j| \leq n \max \left(h, h^{\prime}\right)} .
\end{aligned}
$$

We have,

$$
\begin{aligned}
& \sup _{h \in A_{\epsilon}}\left|\sum_{i=1}^{n} \sum_{j=1}^{i-1}\left(u\left(x_{i}\right)+u\left(x_{j}\right)\right) e_{i, j}(h) \epsilon_{i} \epsilon_{j}\right| \\
& \leq \sup _{h \in A_{\epsilon}}\left|\sum_{i=1}^{n} \sum_{j=1}^{i-1}\left(u\left(x_{i}\right)+u\left(x_{j}\right)\right)\left(e_{i, j}(h)-e_{i, j}\left(h_{n}\right)\right) \epsilon_{i} \epsilon_{j}\right| \\
& +\left|\sum_{i=1}^{n} \sum_{j=1}^{i-1}\left(u\left(x_{i}\right)+u\left(x_{j}\right)\right) e_{i, j}\left(h_{n}\right) \epsilon_{i} \epsilon_{j}\right| .
\end{aligned}
$$

Arguing as in Lemma C.4 of the supplementary material, we have

$$
\limsup _{n \rightarrow \infty}\left\|\sup _{h \in A_{\epsilon}}\left|\sum_{i=1}^{n} \sum_{j=1}^{i-1}\left(u\left(x_{i}\right)+u\left(x_{j}\right)\right)\left(e_{i, j}(h)-e_{i, j}\left(h_{n}\right)\right) \epsilon_{i} \epsilon_{j}\right|\right\|_{p}<\infty,
$$

and by Proposition C.1 of the supplementary material,

$$
\limsup _{n \rightarrow \infty}\left\|\sum_{i=1}^{n} \sum_{j=1}^{i-1}\left(u\left(x_{i}\right)+u\left(x_{j}\right)\right) e_{i, j}\left(h_{n}\right) \epsilon_{i} \epsilon_{j}\right\|_{p}<\infty .
$$

Consequently,

$$
\left.\lim _{n \rightarrow \infty}\left\|\sup _{h \in A_{\epsilon}}\left|\sum_{i=1}^{n} \sum_{j=1}^{i-1}\left(u\left(x_{i}\right)+u\left(x_{j}\right)\right) e_{i, j}(h) \epsilon_{i} \epsilon_{j}\right|\right\|\right|_{p}<\infty .
$$

The limit (4.4) is proved by collecting (4.8), (4.9) and (4.10). 


\subsubsection{Proof of Lemma 4.2}

We can write the following decomposition,

$$
\begin{aligned}
& T_{n}(h)-\mathbb{E}\left(T_{n}(h)\right)=\frac{1}{n} \sum_{i=1}^{n} u\left(x_{i}\right)\left[\left(\hat{r}\left(x_{i}\right)-\mathbb{E}\left(\hat{r}\left(x_{i}\right)\right)\right)^{2}-\mathbb{E}\left[\left(\hat{r}\left(x_{i}\right)-\mathbb{E}\left(\hat{r}\left(x_{i}\right)\right)\right)^{2}\right]\right] \\
& +\frac{2}{n} \sum_{i=1}^{n} u\left(x_{i}\right)\left(\hat{r}\left(x_{i}\right)-\mathbb{E}\left(\hat{r}\left(x_{i}\right)\right)\right)\left(\mathbb{E}\left(\hat{r}\left(x_{i}\right)\right)-r\left(x_{i}\right)\right) \\
& =\sum_{j=1}^{n} C_{j, n}(h) \epsilon_{j}+\sum_{j=1}^{n} \sum_{l=1}^{j-1} B_{j, l}(h) \epsilon_{j} \epsilon_{l}+\sum_{j=1}^{n} D_{j, n}(h)\left(\epsilon_{j}^{2}-\mathbb{E}\left(\epsilon_{j}^{2}\right)\right),
\end{aligned}
$$

where,

$$
\begin{aligned}
C_{j, n}(h) & =\frac{2}{n^{2} h} \sum_{i=1}^{n} u\left(x_{i}\right) K\left(\frac{x_{i}-x_{j}}{h}\right) B\left(x_{i}, h\right), B\left(x_{i}, h\right)=\mathbb{E}\left(\hat{r}\left(x_{i}\right)\right)-r\left(x_{i}\right), \\
B_{j, l}(h) & =\frac{2}{n^{3} h^{2}} \sum_{i=1}^{n} u\left(x_{i}\right) K\left(\frac{x_{i}-x_{j}}{h}\right) K\left(\frac{x_{i}-x_{l}}{h}\right), \\
D_{j, n}(h) & =\frac{1}{n^{3} h^{2}} \sum_{i=1}^{n} u\left(x_{i}\right) K^{2}\left(\frac{x_{i}-x_{j}}{h}\right) .
\end{aligned}
$$

Proof of (4.5). Let

$$
c_{j, n}(h)=n h C_{j, n}(h)=\frac{2}{n} \sum_{i=1}^{n} u\left(x_{i}\right) K\left(\frac{x_{i}-x_{j}}{h}\right) B\left(x_{i}, h\right)
$$

with $B\left(x_{i}, h\right)=\mathbb{E}\left(\hat{r}\left(x_{i}\right)\right)-r\left(x_{i}\right)=\frac{1}{n h} \sum_{l=1}^{n} K\left(\frac{x_{i}-x_{l}}{h}\right) r\left(x_{l}\right)-r\left(x_{i}\right)$. We get, for $h, h^{\prime} \in H_{n}$,

$$
c_{j, n}(h)-c_{j, n}\left(h^{\prime}\right)=\frac{2}{n} \sum_{i=1}^{n} u\left(x_{i}\right)\left(K\left(\frac{x_{i}-x_{j}}{h}\right) B\left(x_{i}, h\right)-K\left(\frac{x_{i}-x_{j}}{h^{\prime}}\right) B\left(x_{i}, h^{\prime}\right)\right) .
$$

Now, since $K$ is a Lipschitz function,

$$
\begin{aligned}
& \left|K\left(\frac{x_{i}-x_{j}}{h}\right) B\left(x_{i}, h\right)-K\left(\frac{x_{i}-x_{j}}{h^{\prime}}\right) B\left(x_{i}, h^{\prime}\right)\right| \\
& \leq \operatorname{cst}\left(\left|B\left(x_{i}, h\right)-B\left(x_{i}, h^{\prime}\right)\right|+\left|x_{i}-x_{j}\right| \sup _{h \in H_{n}}\left|B\left(x_{i}, h\right)\right| \frac{\left|h-h^{\prime}\right|}{h h^{\prime}}\right) \mathbb{I}_{\left|x_{i}-x_{j}\right| \leq \max \left(h, h^{\prime}\right)} .
\end{aligned}
$$


We have, for any $h, h^{\prime} \in H_{n}$,

$$
u\left(x_{i}\right)\left|B\left(x_{i}, h\right)-B\left(x_{i}, h^{\prime}\right)\right| \leq \operatorname{cst} n^{-1 / 5}\left|h-h^{\prime}\right|,
$$

and by the proof of Lemma A.1 in Appendix A.1 of the supplementary material, $\left|B\left(x_{i}, h\right)\right| \leq$ cst $h^{2}$. Hence, for $h, h^{\prime} \in H_{n}$,

$$
\left|c_{j, n}(h)-c_{j, n}\left(h^{\prime}\right)\right| \leq \operatorname{cst}^{-2 / 5}\left|h-h^{\prime}\right|
$$

Since $K$ is compactly supported, we have,

$$
\sup _{h \in H_{n}}\left|c_{j, n}(h)\right| \leq c s t \max _{1 \leq i \leq n} \sup _{h \in H_{n}}\left(h\left|B\left(x_{i}, h\right)\right|\right)=O\left(n^{-\frac{3}{5}}\right) .
$$

Consequently, we obtain using Lemma C.1 of the supplementary material,

$$
\lim _{n \rightarrow \infty}\left\|\sup _{h \in H_{n}} n h\left|\sum_{j=1}^{n} C_{j, n}(h) \epsilon_{j}\right|\right\|_{2}=0 .
$$

Now, let

$$
d_{j, n}(h)=n h D_{j, n}(h)=\frac{1}{n^{2} h} \sum_{i=1}^{n} u\left(x_{i}\right) K^{2}\left(\frac{x_{i}-x_{j}}{h}\right) .
$$

We have, $\left|d_{j, n}(h)\right| \leq \frac{c s t}{n}$ and $\left|d_{j, n}(h)-d_{j, n}\left(h^{\prime}\right)\right| \leq n^{-4 / 5}\left|h-h^{\prime}\right|$. Then Lemma C.2 of the supplementary material gives,

$$
\lim _{n \rightarrow \infty}\left\|\sup _{h \in H_{n}} n h\left|\sum_{j=1}^{n} D_{j, n}(h)\left(\epsilon_{j}^{2}-\mathbb{E}\left(\epsilon_{j}^{2}\right)\right)\right|\right\|_{p}=0 .
$$

Now, let

$$
b_{j, l}(h)=n h B_{j, l}(h)=\frac{1}{n^{2} h} \sum_{i=1}^{n} u\left(x_{i}\right) K\left(\frac{x_{i}-x_{j}}{h}\right) K\left(\frac{x_{i}-x_{l}}{h}\right),
$$

we have,

$$
b_{j, l}^{2}(h)=\frac{1}{n^{4} h^{2}}\left(\sum_{i=1}^{n} u\left(x_{i}\right) K\left(\frac{x_{i}-x_{j}}{h}\right) K\left(\frac{x_{i}-x_{l}}{h}\right)\right)^{2} \leq \frac{c s t}{n^{2}} \mathbb{I}_{|j-l| \leq 2 n h} .
$$


Our purpose now is to control, for $h, h^{\prime} \in H_{n}$, the increment $\left|b_{j, l}(h)-b_{j, l}\left(h^{\prime}\right)\right|$. We have,

$$
\left|b_{j, l}(h)-b_{j, l}\left(h^{\prime}\right)\right| \leq \frac{c s t \max \left(h, h^{\prime}\right)}{n h h^{\prime}}\left|h-h^{\prime}\right| \leq \operatorname{cst} n^{-4 / 5}\left|h-h^{\prime}\right| \mathbb{I}_{|j-l| \leq 2 n h} .
$$

Then by Lemma C.3 of the supplementary material, we obtain

$$
\lim _{n \rightarrow \infty}\left\|\sup _{h \in H_{n}} n h\left|\sum_{j=1}^{n} \sum_{l=1}^{j-1} B_{j, l}(h) \epsilon_{j} \epsilon_{l}\right|\right\|_{p}=0
$$

Collecting (4.11), (4.12), (4.13) and (4.14), we finally deduce (4.5).

Proofs of (4.6) and (4.7). Let us note that the function $h \longmapsto \mathbb{E}\left(T_{n}(h)\right)$ is twice differentiable with continuous second derivative and that for $i \in\{1,2\}$

$\frac{\partial^{i}}{\partial h^{i}} \mathbb{E}\left(T_{n}(h)\right)=\mathbb{E}\left(T_{n}^{(i)}(h)\right)$. We only discuss the proof (4.7) since that of (4.6) is similar. Taking the second derivative over $h$ in (4.11), we have

$T_{n}^{\prime \prime}(h)-\mathbb{E}\left(T_{n}^{\prime \prime}(h)\right)=\sum_{j=1}^{n} C_{j, n}^{\prime \prime}(h) \epsilon_{j}+\sum_{j=1}^{n} \sum_{l=1}^{j-1} B_{j, l}^{\prime \prime}(h) \epsilon_{j} \epsilon_{l}+\sum_{j=1}^{n} D_{j, n}^{\prime \prime}(h)\left(\epsilon_{j}^{2}-\mathbb{E}\left(\epsilon_{j}^{2}\right)\right)$,

where, letting $B\left(x_{i}, h\right)=\mathbb{E}\left(\hat{r}\left(x_{i}\right)\right)-r\left(x_{i}\right)$,

$$
\begin{aligned}
& C_{j, n}^{\prime \prime}(h)=\frac{1}{n^{2} h} \sum_{i=1}^{n} u\left(x_{i}\right)\left(\frac{B\left(x_{i}, h\right)}{h^{2}} G_{1}\left(\frac{x_{i}-x_{j}}{h}\right)+\frac{B^{\prime}\left(x_{i}, h\right)}{h} G_{2}\left(\frac{x_{i}-x_{j}}{h}\right)\right. \\
& \left.+B^{\prime \prime}\left(x_{i}, h\right) G_{3}\left(\frac{x_{i}-x_{j}}{h}\right)\right) \\
& B_{j, l}^{\prime \prime}(h)=\frac{1}{n^{3} h^{4}} \sum_{i=1}^{n} u\left(x_{i}\right) F_{1}\left(\frac{x_{i}-x_{j}}{h}\right) F_{2}\left(\frac{x_{i}-x_{l}}{h}\right) \\
& D_{j, n}^{\prime \prime}(h)=\frac{1}{n^{3} h^{4}} \sum_{i=1}^{n} u\left(x_{i}\right) F\left(\frac{x_{i}-x_{j}}{h}\right),
\end{aligned}
$$

where $F_{1}, F_{2}, F, G_{1}, G_{2}$ and $G_{3}$ are bounded functions of class $C^{1}$, [-1, 1]compactly supported. The proof of (4.7) is analogous to (4.4) and (4.5).

\subsection{Proof of Proposition 3.1}

We have from Lemma 2.1,

$$
\lim _{n \rightarrow \infty} \sup _{h \in H_{n}}\left|\frac{\mathbb{E}\left(T_{n}(h)\right)}{D_{n}(h)}-1\right|=0 .
$$


From this, we claim that $\frac{h_{n}}{h_{n}^{*}}$ converges to 1 , as $n$ tends to infinity. In fact, by the definition of $h_{n}$, it holds $\mathbb{E}\left(T_{n}\left(h_{n}\right)\right) \leq \mathbb{E}\left(T_{n}\left(h_{n}^{*}\right)\right)$. Hence,

$$
D_{n}\left(h_{n}\right) \frac{\mathbb{E}\left(T_{n}\left(h_{n}\right)\right)}{D_{n}\left(h_{n}\right)} \leq \frac{\mathbb{E}\left(T_{n}\left(h_{n}^{*}\right)\right)}{D_{n}\left(h_{n}^{*}\right)} D_{n}\left(h_{n}^{*}\right),
$$

so by (4.15) and the definition of $h_{n}^{*}$, we deduce that, for a fixed $\epsilon>0$ there exists $n_{0}$ such that for any $n \geq n_{0}$,

$$
(1-\epsilon) D_{n}\left(h_{n}\right) \leq(1+\epsilon) D_{n}\left(h_{n}^{*}\right) \leq(1+\epsilon) D_{n}\left(h_{n}\right),
$$

so that $\lim _{n \rightarrow \infty} \frac{D_{n}\left(h_{n}^{*}\right)}{D_{n}\left(h_{n}\right)}=1$, which ensures that $\lim _{n \rightarrow \infty} \frac{h_{n}^{*}}{h_{n}}=1$, in fact (supposing without loss of generality that $\left.D_{n}\left(h_{n}^{*}\right)-D_{n}\left(h_{n}\right) \neq 0\right)$,

$$
\begin{aligned}
& \frac{h_{n}^{*}-h_{n}}{h_{n}}=\frac{h_{n}^{*}-h_{n}}{D_{n}\left(h_{n}^{*}\right)-D_{n}\left(h_{n}\right)} \frac{D_{n}\left(h_{n}^{*}\right)-D_{n}\left(h_{n}\right)}{h_{n}} \\
& =\frac{h_{n}^{*}-h_{n}}{D_{n}\left(h_{n}^{*}\right)-D_{n}\left(h_{n}\right)} \frac{D_{n}\left(h_{n}\right)}{h_{n}}\left(\frac{D_{n}\left(h_{n}^{*}\right)}{D_{n}\left(h_{n}\right)}-1\right)=\frac{D_{n}\left(h_{n}\right)}{h_{n} D_{n}^{\prime}\left(h^{*}\right)}\left(\frac{D_{n}\left(h_{n}^{*}\right)}{D_{n}\left(h_{n}\right)}-1\right),
\end{aligned}
$$

where $h^{*}$ is between $h_{n}$ and $h_{n}^{*}$ which are all in $H_{n}$, consequently $\lim \sup _{n \rightarrow \infty}\left|\frac{D_{n}\left(h_{n}\right)}{h_{n} D_{n}^{\prime}\left(h^{*}\right)}\right|<$ $\infty$ and then the behavior of $\frac{h_{n}^{*}-h_{n}}{h_{n}}$ is deduced from the fact that $\frac{D_{n}\left(h_{n}^{*}\right)}{D_{n}\left(h_{n}\right)}-1$ tends to 0 as $n$ tends to infinity.

In order to complete the proof of Proposition 3.1, we only need to prove that both $\frac{D_{n}\left(\hat{h}_{n}\right)}{D_{n}\left(h_{n}\right)}$ and $\frac{D_{n}\left(\hat{h}_{M}\right)}{D_{n}\left(h_{n}\right)}$ converge in probability to 1 as $n$ tends to infinity (recall that both $\hat{h}_{M}$ and $\hat{h}_{n}$ belong to $H_{n}$ ). We refer the reader to Rice, J. (1984) for similar arguments. For this, we have to prove an analogous to the limit (4.15),

$$
\lim _{n \rightarrow \infty}\left\|\sup _{h \in H_{n}}\left|\frac{T_{n}(h)}{D_{n}(h)}-1\right|\right\|_{p}=0, \text { for some } p>8
$$

which gives, from the same previous arguments, that, for any $\epsilon>0$,

$$
\lim _{n \rightarrow \infty} \mathbb{P}\left((1-\epsilon) \leq(1+\epsilon) \frac{D_{n}\left(h_{n}^{*}\right)}{D_{n}\left(\hat{h}_{n}\right)} \leq(1+\epsilon)\right)=1 .
$$

Since $\inf _{h \in H_{n}} n h D_{n}(h)>0$ and by (4.15), the limit (4.16) is proved as soon as,

$$
\lim _{n \rightarrow \infty}\left\|\sup _{h \in H_{n}} n h\left|T_{n}(h)-\mathbb{E}\left(T_{n}(h)\right)\right|\right\|_{p}=0
$$


which immediately follows from Lemma 4.2 (more precisely (4.5) of Subsection 4.1). Our purpose now is to prove that $\frac{\hat{h}_{M}}{h_{n}}$ converges in probability to 1 as $n$ tends to infinity. Recall that $\mathrm{CL}(h)=T_{n}(h)+\delta_{2}(h)+n^{-1}\left\|U^{1 / 2}(Y-r)\right\|^{2}$, where $\delta_{2}(h)=2 n^{-1}(Y-r)^{\prime} U(r-\hat{r})+2 \sigma^{2} n^{-1} \operatorname{tr}(U R L)$. We have, using Lemma 4.1 of Subsection 4.1,

$$
\lim _{n \rightarrow \infty}\left\|\sup _{h \in H_{n}} n h\left|\delta_{2}(h)\right|\right\|_{p}=0
$$

or equivalently, since $\inf _{h \in H_{n}} n h D_{n}(h)>0$,

$$
\lim _{n \rightarrow \infty}\left\|\sup _{h \in H_{n}}\left|\frac{\delta_{2}(h)}{D_{n}(h)}\right|\right\|_{p}=0 .
$$

This last limit, together with (4.16), give

$$
\lim _{n \rightarrow \infty} \|\left.\sup _{h \in H_{n}}\left|\frac{T_{n}(h)+\delta_{2}(h)}{D_{n}(h)}-1\right|\right|_{p}=0 .
$$

Now, we have, since $n^{-1}\left\|U^{1 / 2}(Y-r)\right\|^{2}$ doesn't depend on $h$,

$$
\hat{h}_{M} \in \operatorname{argmin}_{h \in H_{n}}\left(T_{n}(h)+\delta_{2}(h)\right),
$$

so that using (4.17) and the same previous arguments, we prove that

$$
\frac{D_{n}\left(\hat{h}_{M}\right)}{D_{n}\left(h_{n}\right)} \rightarrow 1 \text {, in probability as } n \rightarrow \infty \text {. }
$$

It remains to prove the statement concerning the fourth ratio $\hat{h}_{G} / h_{n}$. A way to do it is to appeal to the a.o. of CL that we have proved above and to show that the difference $G_{X}(h)-\mathrm{CL}(h)$ is uniformly negligible as compared to $\mathbb{E}\left(T_{n}(h)\right)$. Using that $\operatorname{tr}(U R L)=\sum_{i=1}^{n} u\left(x_{i}\right) t(h)$ where $t(h)=$ $n^{-1} h^{-1} K(0)$, we have from the definition (2.6) of $G_{X}(h)$ and the property $\Xi_{\mathrm{X}}(t)=1+2 t+O\left(t^{2}\right)$

$$
\begin{gathered}
\frac{n}{\sum_{i=1}^{n} u\left(x_{i}\right)}\left(G_{X}(h)-\mathrm{CL}(h)\right) \\
=\frac{1}{\sum_{i=1}^{n} u\left(x_{i}\right)}\left\|U^{1 / 2}(I-L) Y\right\|^{2} \times \Xi_{\mathrm{X}}(t(h))-\left(\frac{1}{\sum_{i=1}^{n} u\left(x_{i}\right)}\left\|U^{1 / 2}(I-L) Y\right\|^{2}+2 \sigma^{2} t(h)\right)
\end{gathered}
$$




$$
=\hat{\sigma}_{h}^{2} \times\left(\Xi_{\mathrm{X}}(t(h))-1\right)-2 \sigma^{2} t(h)=\left(\hat{\sigma}_{h}^{2}-\sigma^{2}\right)(2 t(h))+\hat{\sigma}_{h}^{2} O(t(h))^{2} .
$$

Clearly this last quantity is $o_{P}\left(n^{-1} h^{-1}\right)$ uniformly over a domain of $h$ where $n h \rightarrow \infty$ as soon as $\hat{\sigma}_{h}^{2}$ converges toward $\sigma^{2}$ uniformly over this domain. To see that this is true for the domain $H_{n}$ under our assumptions, let us decompose

$$
\begin{aligned}
& \frac{\sum_{i=1}^{n} u\left(x_{i}\right)}{n} \hat{\sigma}_{h}^{2} \\
& =\mathbb{E} T_{n}(h)+\left(T_{n}(h)-\mathbb{E} T_{n}(h)\right)+\delta_{2}(h)+\frac{1}{n}\left\|U^{1 / 2}(Y-r)\right\|^{2}-2 \sigma^{2} \frac{\sum_{i=1}^{n} u\left(x_{i}\right)}{n} t(h),
\end{aligned}
$$

(directly obtained by combining (2.4) and (2.5)). Now the required convergence is a consequence of Lemma 2.1, the limit (4.5) in Lemma 4.2, the limit (4.1) in Lemma 4.1 and the fact that $\frac{1}{\sum_{i=1}^{n} u\left(x_{i}\right)}\left\|U^{1 / 2}(Y-r)\right\|^{2} \rightarrow \sigma^{2}$ in probability under Conditions (C). The proof of Proposition 3.1 is completed.

\subsection{Proof of Theorem 3.1}

The following lemma is crucial for the proof of Theorem 3.1. It gives conditions under which $v_{n}\left(\hat{h}_{M}-\hat{h}_{n}\right)$ and $v_{n}\left(\hat{h}_{M}-\hat{h}_{n}\right)$ converge to a normal law with some rate $v_{n}$. Its proof is given in Section $\mathbf{A . 3}$ of the supplementary material.

Lemma 4.3. If, as $n$ tends to infinity and for some positive rate $a_{n}$, (recall that $\delta_{2}(h)$ is defined in Equation (2.5)),

1. $a_{n} \delta_{2}^{\prime}\left(h_{n}\right)$ converges to a centered normal law with variance $V$,

2. $a_{n}\left(\delta_{2}^{\prime}\left(h_{n}\right)-\delta_{2}^{\prime}\left(\hat{h}_{n}\right)\right)$ converges in probability to 0 ,

3. $\frac{\mathrm{CL}^{\prime \prime}\left(h^{*}\right)}{\mathrm{E}\left(T_{n}^{\prime \prime}\left(h_{n}\right)\right)}$ tends in probability to 1 for any $h^{*}$ between $\hat{h}_{n}$ and $\hat{h}_{M}$,

then

$$
a_{n} \mathbb{E}\left(T_{n}^{\prime \prime}\left(h_{n}\right)\right)\left(\hat{h}_{n}-\hat{h}_{M}\right)
$$

converges in distribution to a centered normal law with variance $V$.

Furthermore, implicitly defining $\delta_{3}(h)$ by $G_{X}(h)=T_{n}(h)+\delta_{3}(h)+n^{-1} \| U^{1 / 2}(Y-$ $r) \|^{2}$, if, in addition to the 3 steps above, we show that

4. $a_{n}\left(\delta_{3}^{\prime}(h)-\delta_{2}^{\prime}(h)\right)$ converges in probability to 0 , uniformly over $H_{n}$ 
5. $\frac{G_{X}^{\prime \prime}\left(h^{*}\right)}{\mathbb{E}\left(T_{n}^{\prime \prime}\left(h_{n}\right)\right)}$ tends in probability to 1 for any $h^{*}$ between $\hat{h}_{n}$ and $\hat{h}_{G}$, then

$$
a_{n} \mathbb{E}\left(T_{n}^{\prime \prime}\left(h_{n}\right)\right)\left(\hat{h}_{n}-\hat{h}_{G}\right)
$$

converges in distribution to the same centered normal law.

According to Lemma 4.3, we have to consider five steps. We study each of them in the following five subsections. The final subsection concludes the proof of Theorem 3.1.

\subsubsection{Step 1: convergence in distribution of $a_{n} \delta_{2}^{\prime}\left(h_{n}\right)$.}

The following proposition studies the asymptotic distribution of $a_{n} \delta_{2}^{\prime}\left(h_{n}\right)$ for $a_{n}=\sqrt{\frac{n}{h_{n}^{2}}}$.

Proposition 4.1. Suppose that the assumptions of Theorem 3.1 are satisfied. Then the following two assertions are equivalent.

- $\sqrt{\frac{n}{h_{n}^{2}}} \delta_{2}^{\prime}\left(h_{n}\right)$ converges in distribution as $n$ tends to infinity to a centered normal law with variance $4 \mathrm{~V}$

- $\sqrt{\frac{n}{h_{n}^{2}}} \sum_{i=1}^{n}\left(\tilde{a}_{i, n}\left(h_{n}\right) u\left(x_{i}\right) \epsilon_{i}+\sum_{j=1}^{i-1}\left(u\left(x_{i}\right)+u\left(x_{j}\right)\right) b_{i, j}\left(h_{n}\right) \epsilon_{i} \epsilon_{j}\right)$ converges to a centered normal law with variance $V$, where

$$
\tilde{a}_{i, n}\left(h_{n}\right)=-C_{K} \frac{h_{n}}{n} r^{\prime \prime}\left(x_{i}\right)
$$

Proof of Proposition 4.1. Recall that, for $G(u)=-u K^{\prime}(u)$, for any $h \in] 0, \epsilon[$ and any $n \geq 1$,

$$
\begin{aligned}
& \delta_{2}^{\prime}(h)=2 \sum_{i=1}^{n} a_{i}(h) u\left(x_{i}\right) \epsilon_{i}+2 \sum_{i=1}^{n} \sum_{j=1}^{i-1}\left(u\left(x_{i}\right)+u\left(x_{j}\right)\right) b_{i, j}(h) \epsilon_{i} \epsilon_{j} \\
& +2 \sum_{i=1}^{n} b_{i, i}(h) u\left(x_{i}\right)\left(\epsilon_{i}^{2}-\mathbb{E}\left(\epsilon_{i}^{2}\right)\right),
\end{aligned}
$$

where,

$$
a_{i}(h)=-\frac{1}{n} \frac{\partial}{\partial h} \mathbb{E}\left(\hat{r}\left(x_{i}\right)\right)=\frac{1}{n^{2} h^{2}} \sum_{j=1}^{n}(K-G)\left(\frac{x_{i}-x_{j}}{h}\right) r\left(x_{j}\right),
$$




$$
b_{i, j}(h)=\frac{1}{n^{2} h^{2}} K\left(\frac{x_{i}-x_{j}}{h}\right)-\frac{1}{n^{2} h^{2}} G\left(\frac{x_{i}-x_{j}}{h}\right) \text { and } b_{i, i}(h)=\frac{1}{n^{2} h^{2}} K(0) .
$$

We also need, for the proof of Proposition 4.1, the following two lemmas.

Lemma 4.4. Recall that $h_{n}=c n^{-1 / 5}$ and suppose that $\sum_{j=1}^{\infty}\left|\operatorname{Cov}\left(\epsilon_{1}^{2}, \epsilon_{j}^{2}\right)\right|<$ $\infty$, then

$$
\lim _{n \rightarrow \infty} \frac{n}{h_{n}^{2}} \operatorname{Var}\left(\sum_{i=1}^{n} u\left(x_{i}\right) b_{i, i}\left(h_{n}\right)\left(\epsilon_{i}^{2}-\mathbb{E}\left(\epsilon_{i}^{2}\right)\right)\right)=0 .
$$

Proof of Lemma 4.4. We have,

$$
\begin{aligned}
& \frac{n}{h_{n}^{2}} \operatorname{Var}\left(\sum_{i=1}^{n} u\left(x_{i}\right) b_{i, i}\left(h_{n}\right)\left(\epsilon_{i}^{2}-\mathbb{E}\left(\epsilon_{i}^{2}\right)\right)\right)=\frac{n}{h_{n}^{2} n^{4} h_{n}^{4}} K^{2}(0) \sum_{i=1}^{n} \sum_{j=1}^{n} u\left(x_{i}\right) u\left(x_{j}\right) \operatorname{Cov}\left(\epsilon_{i}^{2}, \epsilon_{j}^{2}\right) \\
& \leq \frac{1}{n^{2} h_{n}^{6}} K^{2}(0)\|u\|_{\infty}^{2} \sup _{i} \sum_{j=1}^{\infty}\left|\operatorname{Cov}\left(\epsilon_{i}^{2}, \epsilon_{j}^{2}\right)\right| .
\end{aligned}
$$

The proof of this lemma is achieved since $\lim _{n \rightarrow \infty} n^{2} h_{n}^{6}=\lim _{n \rightarrow \infty} n^{4 / 5}=\infty$.

Lemma 4.5. Recall that $h_{n}=c n^{-1 / 5}$. We have, noting $C_{K}=\int x^{2} K(x) d x$,

$$
\lim _{n \rightarrow \infty} \frac{n}{h_{n}^{2}} \operatorname{Var}\left(\sum_{i=1}^{n}\left(a_{i}\left(h_{n}\right)+C_{K} \frac{h_{n}}{n} r^{\prime \prime}\left(x_{i}\right)\right) u\left(x_{i}\right) \epsilon_{i}\right)=0 .
$$

Proof of Lemma 4.5. Clearly, we have using Lemma A.3 of Appendix A.2 of the supplementary material,

$$
a_{i}\left(h_{n}\right)+C_{K} \frac{h_{n}}{n} r^{\prime \prime}\left(x_{i}\right)=O\left(\frac{h_{n}^{2}}{n}+\frac{1}{n^{2} h_{n}^{3}}\right),
$$

and

$$
\begin{aligned}
& \operatorname{Var}\left(\sum_{i=1}^{n}\left(a_{i}\left(h_{n}\right)+C_{K} \frac{h_{n}}{n} r^{\prime \prime}\left(x_{i}\right)\right) u\left(x_{i}\right) \epsilon_{i}\right) \\
& =\sum_{i=1}^{n} \sum_{j=1}^{n}\left(a_{i}\left(h_{n}\right)+C_{K} \frac{h_{n}}{n} r^{\prime \prime}\left(x_{i}\right)\right)\left(a_{j}\left(h_{n}\right)+C_{K} \frac{h_{n}}{n} r^{\prime \prime}\left(x_{j}\right)\right) u\left(x_{i}\right) u\left(x_{j}\right) \operatorname{Cov}\left(\epsilon_{i}, \epsilon_{j}\right) \\
& \leq \operatorname{cst} \sup _{i}\left(\left(a_{i}\left(h_{n}\right)+C_{K} \frac{h_{n}}{n} r^{\prime \prime}\left(x_{i}\right)\right) u\left(x_{i}\right)\right)^{2} n \sigma^{2} \\
& =O\left(n\left(\frac{h_{n}^{2}}{n}+\frac{1}{n^{2} h_{n}^{3}}\right)^{2}\right)=O\left(n^{-9 / 5}\right)=o\left(\frac{h_{n}^{2}}{n}\right) .
\end{aligned}
$$


The proof of Lemma 4.5 is complete.

End of the proof of Proposition 4.1. We have, using (4.18),

$$
\begin{aligned}
& \sqrt{\frac{n}{h_{n}^{2}}} \delta_{2}^{\prime}\left(h_{n}\right)=2 \sqrt{\frac{n}{h_{n}^{2}}} \sum_{i=1}^{n}\left(\tilde{a}_{i, n}\left(h_{n}\right) u\left(x_{i}\right) \epsilon_{i}+\sum_{j=1}^{i-1}\left(u\left(x_{i}\right)+u\left(x_{j}\right)\right) b_{i, j}\left(h_{n}\right) \epsilon_{i} \epsilon_{j}\right) \\
& +2 \sqrt{\frac{n}{h_{n}^{2}}} \sum_{i=1}^{n}\left(a_{i}\left(h_{n}\right)-\tilde{a}_{i, n}\left(h_{n}\right)\right) u\left(x_{i}\right) \epsilon_{i}+2 \sqrt{\frac{n}{h_{n}^{2}}} \sum_{i=1}^{n} b_{i, i}\left(h_{n}\right) u\left(x_{i}\right)\left(\epsilon_{i}^{2}-\mathbb{E}\left(\epsilon_{i}^{2}\right)\right) .
\end{aligned}
$$

The proof of Proposition 4.1 is complete if $\sqrt{\frac{n}{h_{n}^{2}}} \sum_{i=1}^{n}\left(a_{i}\left(h_{n}\right)-\tilde{a}_{i, n}\left(h_{n}\right)\right) u\left(x_{i}\right) \epsilon_{i}$ and $\sqrt{\frac{n}{h_{n}^{2}}} \sum_{i=1}^{n} b_{i, i}\left(h_{n}\right) u\left(x_{i}\right)\left(\epsilon_{i}^{2}-\mathbb{E}\left(\epsilon_{i}^{2}\right)\right)$ converge in probability to 0 as $n$ tends to infinity, which are satisfied due to Lemmas 4.4 and 4.5.

\subsubsection{Step 2: convergence in probability of $a_{n}\left(\delta_{2}^{\prime}\left(h_{n}\right)-\delta_{2}^{\prime}\left(\hat{h}_{n}\right)\right)$}

The following proposition checks step 2 of Lemma 4.3.

Proposition 4.2. Under the assumptions of Theorem 3.1, $\sqrt{\frac{n}{h_{n}^{2}}}\left(\delta_{2}^{\prime}\left(h_{n}\right)-\right.$ $\left.\delta_{2}^{\prime}\left(\hat{h}_{n}\right)\right)$ converges in probability to 0 as $n$ tends to infinity.

Proof of Proposition 4.2. We have,

$$
\sqrt{\frac{n}{h_{n}^{2}}}\left(\delta_{2}^{\prime}\left(h_{n}\right)-\delta_{2}^{\prime}\left(\hat{h}_{n}\right)\right)=\sqrt{\frac{n}{h_{n}^{2}}}\left(h_{n}-\hat{h}_{n}\right) \delta_{2}^{\prime \prime}\left(h^{*}\right)=\sqrt{n}\left(1-\frac{\hat{h}_{n}}{h_{n}}\right) \delta_{2}^{\prime \prime}\left(h^{*}\right),
$$

where $h^{*}$ is an element of $H_{n}$ between $h_{n}$ and $\hat{h}_{n}$ and since $\hat{h}_{n} / h_{n}$ converges in probability to 1 as $n$ tends to infinity (by Proposition 3.1), we deduce that

$$
\lim _{n \rightarrow \infty} \mathbb{P}\left(h^{*} \notin A_{\epsilon}\right)=0, \quad \forall \epsilon>0
$$

where, for fixed $\epsilon>0, A_{\epsilon}=\left\{h \in H_{n},\left|\frac{h}{h_{n}}-1\right| \leq \epsilon\right\}$. Now, we have for any 


$$
\begin{aligned}
M & > \\
& \mathbb{P}\left(a_{n}\left|\delta_{2}^{\prime}\left(h_{n}\right)-\delta_{2}^{\prime}\left(\hat{h}_{n}\right)\right| \geq \epsilon^{2}\right) \\
& \leq \mathbb{P}\left(\sqrt{n}\left|1-\frac{\hat{h}_{n}}{h_{n}}\right| \delta_{2}^{\prime \prime}\left(h^{*}\right) \mid \geq \epsilon^{2}, h^{*} \in A_{\epsilon}\right)+\mathbb{P}\left(\sqrt{n}\left(1-\frac{\hat{h}_{n}}{h_{n}}\right)\left|\delta_{2}^{\prime \prime}\left(h^{*}\right)\right| \geq \epsilon^{2}, h^{*} \notin A_{\epsilon}\right) \\
& \leq \mathbb{P}\left(\sqrt{n}\left|1-\frac{\hat{h}_{n}}{h_{n}}\right|\left|\delta_{2}^{\prime \prime}\left(h^{*}\right)\right| \mathbb{I}_{h^{*} \in A_{\epsilon}} \geq \epsilon^{2}\right)+\mathbb{P}\left(h^{*} \notin A_{\epsilon}\right) \\
& \leq \mathbb{P}\left(\sqrt{n}\left|1-\frac{\hat{h}_{n}}{h_{n}}\right| \sup _{h \in A_{\epsilon}}\left|\delta_{2}^{\prime \prime}(h)\right| \geq \epsilon^{2}\right)+\mathbb{P}\left(h^{*} \notin A_{\epsilon}\right) \\
& \leq \mathbb{P}\left(\sqrt{n}\left|1-\frac{\hat{h}_{n}}{h_{n}}\right| \sup _{h \in A_{\epsilon}}\left|\delta_{2}^{\prime \prime}(h)\right| \geq \epsilon^{2}, \sqrt{n} \sup _{h \in A_{\epsilon}}\left|\delta_{2}^{\prime \prime}(h)\right| \geq M\right) \\
& +\mathbb{P}\left(\sqrt{n}\left|1-\frac{\hat{h}_{n}}{h_{n}}\right| \sup _{h \in A_{\epsilon}}\left|\delta_{2}^{\prime \prime}(h)\right| \geq \epsilon^{2}, \sqrt{n} \sup _{h \in A_{\epsilon}}\left|\delta_{2}^{\prime \prime}(h)\right|<M\right)+\mathbb{P}\left(h^{*} \notin A_{\epsilon}\right) \\
& \leq \mathbb{P}\left(\sqrt{n} \sup _{h \in A_{\epsilon}}\left|\delta_{2}^{\prime \prime}(h)\right| \geq M\right)+\mathbb{P}\left(M\left|1-\frac{\hat{h}_{n}}{h_{n}}\right| \geq \epsilon^{2}\right)+\mathbb{P}\left(h^{*} \notin A_{\epsilon}\right),
\end{aligned}
$$

which tends to 0 by letting first $n$ tends to infinity and then $M$ tends to infinity, due to Proposition 3.1, (4.4) and (4.19).

\subsubsection{Step 3: convergence in probability of $\frac{\mathrm{CL}^{\prime \prime}\left(h^{*}\right)}{\mathbb{E}\left(T_{n}^{\prime \prime}\left(h_{n}\right)\right)}$}

Proposition 4.3. Under the assumptions of Theorem 3.1, $\frac{\mathrm{CL}^{\prime \prime}\left(h^{*}\right)}{\mathbb{E}\left(T_{n}^{\prime \prime}\left(h_{n}\right)\right)}$ tends in probability to 1 , as $n \rightarrow \infty$, for any $h^{*}$ between $\hat{h}_{n}$ and $\hat{h}_{M}$.

Proof of Proposition 4.3. We have, for any $h>0, \mathrm{CL}^{\prime \prime}(h)=T_{n}^{\prime \prime}(h)+\delta_{2}^{\prime \prime}(h)$, and

$$
\frac{\mathrm{CL}^{\prime \prime}(h)}{\mathbb{E}\left(T_{n}^{\prime \prime}(h)\right)}=\frac{T_{n}^{\prime \prime}(h)}{\mathbb{E}\left(T_{n}^{\prime \prime}(h)\right)}+\frac{\delta_{2}^{\prime \prime}(h)}{\mathbb{E}\left(T_{n}^{\prime \prime}(h)\right)} .
$$

Our first purpose is to prove that, $\sup _{h \in H_{n}} \frac{\left|\delta_{2}^{\prime \prime}(h)\right|}{\left|\mathbb{E}\left(T_{n}^{\prime \prime}(h)\right)\right|}$ converges to 0 , in probability, as $n$ tends to infinity. Since $\sup _{h \in H_{n}}\left|\frac{D_{n}^{\prime \prime}(h)}{\left|\mathbb{E}\left(T_{n}^{\prime \prime}(h)\right)\right|}-1\right|$ converges to 0 as 
$n$ tends to infinity, with

$D_{n}^{\prime \prime}(h)=3 h^{2} \int_{0}^{1} u(x) r^{\prime \prime 2}(x) d x \int_{-1}^{1} t^{2} K(t) d t+\frac{2}{n h^{3}}\left(\int_{0}^{1} u(x) d x\right) \int_{-1}^{1} K^{2}(y) d y \sigma^{2}$,

(see Lemma A.4 of Appendix A.2 of the supplementary material) it remains then to prove that, $\left(\right.$ since $\inf _{h \in H_{n}} n h^{3}\left|D_{n}^{\prime \prime}(h)\right|>0$ ),

$$
\sup _{h \in H_{n}}\left(n h^{3}\left|\delta_{2}^{\prime \prime}(h)\right|\right) \longrightarrow 0, \text { in probability as } n \rightarrow \infty,
$$

which is proved due to Lemma 4.1 of Subsection 4.1. It remains to prove that

$$
\sup _{h \in H_{n}}\left|\frac{T_{n}^{\prime \prime}(h)}{\mathbb{E}\left(T_{n}^{\prime \prime}(h)\right)}-1\right| \longrightarrow 0, \quad \text { in probability as } n \rightarrow \infty
$$

or equivalently,

$$
\sup _{h \in H_{n}} n h^{3}\left|T_{n}^{\prime \prime}(h)-\mathbb{E}\left(T_{n}^{\prime \prime}(h)\right)\right| \longrightarrow 0, \text { in probability as } n \rightarrow \infty,
$$

which is proved due to Lemma 4.2 of Subsection 4.1. Consequently,

$$
\sup _{h \in H_{n}}\left|\frac{\mathrm{CL}^{\prime \prime}(h)}{\mathbb{E}\left(T_{n}^{\prime \prime}(h)\right)}-1\right| \longrightarrow 0, \text { in probability as } n \rightarrow \infty,
$$

Finally,

$$
\left|\frac{\mathrm{CL}^{\prime \prime}\left(h^{*}\right)}{D_{n}^{\prime \prime}\left(h_{n}\right)}-1\right| \leq \text { cst } \sup _{h \in H_{n}}\left|\frac{\mathrm{CL}^{\prime \prime}(h)}{D_{n}^{\prime \prime}(h)}-1\right|+\left|\frac{D_{n}^{\prime \prime}\left(h^{*}\right)}{D_{n}^{\prime \prime}\left(h_{n}\right)}-1\right| .
$$

By definition of $h^{*}$ and by Proposition 3.1, we deduce that

$$
\left|\frac{D_{n}^{\prime \prime}\left(h^{*}\right)}{D_{n}^{\prime \prime}\left(h_{n}\right)}-1\right| \longrightarrow 0 \text { in probability as } n \rightarrow \infty
$$

and then

$$
\left|\frac{\mathrm{CL}^{\prime \prime}\left(h^{*}\right)}{D_{n}^{\prime \prime}\left(h_{n}\right)}-1\right| \longrightarrow 0 \text { in probability as } n \rightarrow \infty
$$


4.3.4 Step 4: uniform convergence in probability, over $H_{n}$, of $a_{n}\left(\delta_{3}^{\prime}(h)-\delta_{2}^{\prime}(h)\right)$, where $\delta_{3}$ is defined in Lemma 4.3

The following proposition checks step 4 of Lemma 4.3 for $a_{n}=n^{7 / 10}$.

Proposition 4.4. Under the assumptions of Theorem 3.1, $n^{7 / 10}\left(\delta_{3}^{\prime}(h)-\right.$ $\left.\delta_{2}^{\prime}(h)\right)$ converges in probability to 0 uniformly over $H_{n}$ as $n$ tends to infinity.

Proof of Proposition 4.4. From the definition of $G_{\mathrm{X}}$ and the two expressions (2.4), (2.5) of CL, we have

$$
\begin{aligned}
& G_{\mathrm{X}}^{\prime}(h)=\frac{-K(0)}{n h^{2}} \Xi_{\mathrm{X}}^{\prime}\left(\frac{K(0)}{n h}\right) \times\left(n^{-1}\left\|U^{1 / 2}(I-L) Y\right\|^{2}\right) \\
& \quad+\Xi_{\mathrm{X}}\left(\frac{K(0)}{n h}\right) \times \frac{\mathrm{d}}{\mathrm{d} h}\left(n^{-1}\left\|U^{1 / 2}(I-L) Y\right\|^{2}\right) \\
& =\frac{-K(0)}{n h^{2}} \Xi_{\mathrm{X}}^{\prime}\left(\frac{K(0)}{n h}\right)\left(T_{n}(h)+\delta_{2}(h)+n^{-1}\left\|U^{1 / 2}(Y-r)\right\|^{2}-2 \sigma^{2} \frac{\left.\sum_{i=1}^{n} u\left(x_{i}\right)\right)}{n} \frac{K(0)}{n h}\right) \\
& \quad+\Xi_{\mathrm{X}}\left(\frac{K(0)}{n h}\right)\left(T_{n}^{\prime}(h)+\delta_{2}^{\prime}(h)-2 \sigma^{2} \frac{\left.\sum_{i=1}^{n} u\left(x_{i}\right)\right)}{n}\left(-\frac{K(0)}{n h^{2}}\right)\right) .
\end{aligned}
$$

Let,

$$
\begin{aligned}
& I_{1}:=\frac{-K(0)}{n h^{2}} \Xi_{\mathrm{X}}^{\prime}\left(\frac{K(0)}{n h}\right)\left(T_{n}(h)+\delta_{2}(h)\right) \\
& I_{2}:=\left(\Xi_{\mathrm{X}}\left(\frac{K(0)}{n h}\right)-1\right)\left(T_{n}^{\prime}(h)+\delta_{2}^{\prime}(h)\right)
\end{aligned}
$$

and

$$
\begin{aligned}
I_{3}:= & \frac{-K(0)}{n h^{2}} \Xi_{\mathrm{X}}^{\prime}\left(\frac{K(0)}{n h}\right)\left(n^{-1}\left\|U^{1 / 2}(Y-r)\right\|^{2}\right) \\
& +\Xi_{\mathrm{X}}\left(\frac{K(0)}{n h}\right)\left(2 \sigma^{2} \frac{\left.\sum_{i=1}^{n} u\left(x_{i}\right)\right)}{n} \frac{K(0)}{n h^{2}}\right) .
\end{aligned}
$$

From the above expression of $G_{\mathrm{X}}^{\prime}$ we can write (since, by definition $\delta_{3}^{\prime}(h)=$ $\left.G_{\mathrm{X}}^{\prime}(h)-T_{n}^{\prime}(h)\right)$

$$
\delta_{3}^{\prime}(h)-\delta_{2}^{\prime}(h)=I_{1}+I_{2}+I_{3}+2 \sigma^{2} \frac{K(0)}{n h^{2}} \Xi_{\mathrm{X}}^{\prime}\left(\frac{K(0)}{n h}\right) \frac{\left.\sum_{i=1}^{n} u\left(x_{i}\right)\right)}{n} \frac{K(0)}{n h}
$$


The least term of this decomposition is clearly $o\left(n^{-7 / 10}\right)$ for $h$ in $H_{n}$ since $\Xi_{\mathrm{X}}^{\prime}(t)$ is bounded near 0 . We are going to show that this is also true, in probability, uniformly over $H_{n}$, for the first three terms. Let us begin with $I_{3}$. By

rewriting the factor $\Xi_{\mathrm{X}}\left(\frac{K(0)}{n h}\right)=(1 / 2) \Xi_{\mathrm{X}}^{\prime}\left(\frac{K(0)}{n h}\right)+\left(\Xi_{\mathrm{X}}\left(\frac{K(0)}{n h}\right)-(1 / 2) \Xi_{\mathrm{X}}^{\prime}\left(\frac{K(0)}{n h}\right)\right)$ in the second term (4.23) of $I_{3}$ and invoking the required properties of $\Xi_{\mathrm{X}}$ near 0 (recall that $\Xi_{\mathrm{X}}^{\prime}(t)=2+O(t)$ ) then

$$
I_{3}=-\frac{K(0)}{n h^{2}} \Xi_{\mathrm{X}}^{\prime}\left(\frac{K(0)}{n h}\right)\left(n^{-1}\left\|U^{1 / 2}(Y-r)\right\|^{2}-\sigma^{2} \frac{\left.\sum_{i=1}^{n} u\left(x_{i}\right)\right)}{n}\right)+o\left(n^{-2} h^{-3}\right) .
$$

Now, similarly as in the proof of Lemma 4.4, it can be checked that $n^{-1} \| U^{1 / 2}(Y-$ $r) \|^{2}-\sigma^{2} \frac{\left.\sum_{i=1}^{n} u\left(x_{i}\right)\right)}{n}$ is $o_{P}\left(n^{-1 / 10}\right)$, and thus $I_{3}$ is uniformly $o_{P}\left(n^{-7 / 10}\right)$. For $I_{1}$ and $I_{2}$, a such uniform rate results from (4.1), (4.2), (4.5), (4.6) used in a classical way via Markov inequality, and of the stated properties of $D_{n}(h)$ and $D_{n}^{\prime}(h)$.

\subsubsection{Step 5: convergence in probability of $\frac{G_{\mathrm{X}}^{\prime \prime}\left(h^{*}\right)}{\mathbb{E}\left(T_{n}^{\prime \prime}\left(h_{n}\right)\right)}$}

We simply check this step by invoking the above Step 3, and using the following result, where $\delta_{3}$ is defined in Lemma 4.3, whose proof is postponed to the supplementary material (see its Section A.4) .

Proposition 4.5. Under the assumptions of Theorem 3.1, $n^{2 / 5}\left(\delta_{3}^{\prime \prime}(h)-\delta_{2}^{\prime \prime}(h)\right)$ tends in probability to 0 , as $n \rightarrow \infty$, uniformly over $H_{n}$.

\subsubsection{End of the proof of Theorem 3.1}

For the first part of this Theorem we have to check the first three items of Lemma 4.3. We have, from Proposition B.2 of the supplementary material,

$$
n^{7 / 10} \sum_{i=1}^{n}\left(\tilde{a}_{i, n}\left(h_{n}\right) u\left(x_{i}\right) \epsilon_{i}+\sum_{j=1}^{i-1}\left(u\left(x_{i}\right)+u\left(x_{j}\right)\right) b_{i, j}\left(h_{n}\right) \epsilon_{i} \epsilon_{j}\right) \Longrightarrow \mathcal{N}(0, V) .
$$

It follows from Proposition 4.1 that

$$
n^{7 / 10} \delta_{2}^{\prime}\left(h_{n}\right) \Longrightarrow \mathcal{N}(0,4 V)
$$


where $V=c^{2} C_{K}^{2} \sigma^{2} \int_{0}^{1} u^{2}(x) r^{\prime \prime 2}(x) d x+\frac{4}{c^{3}} \sigma^{4} \int_{0}^{1} u^{2}(x) d x \int_{0}^{1}(K-G)^{2}(u) d u$. The two other items of Lemma 4.3 are satisfied using Propositions 4.2 and 4.3. The proof of the first part of Theorem 3.1 is complete using Lemma 4.3 together with the fact that (see Lemma A.4 of the supplementary material), $\mathbb{E}\left(T_{n}^{\prime \prime}\left(h_{n}\right)\right)$ is equivalent to

$n^{-2 / 5}\left(3 c^{2} \int_{0}^{1} u(x) r^{\prime \prime 2}(x) d x\left(\int_{-1}^{1} t^{2} K(t) d t\right)^{2}+\frac{2}{c^{3}}\left(\int_{0}^{1} u(x) d x\right) \int_{-1}^{1} K^{2}(y) d y \sigma^{2}\right)$.

As to the second part, arguing as above, it is now sufficient to observe that the fourth and fifth items of Lemma 4.3 are also satisfied using Propositions 4.4 and 4.5 .

\section{Acknowledgements}

We thank the Editor Marcus Reiss, the Associate Editor and two reviewers for their accurate and constructive comments. This led to a significant improvement of the original manuscript, in particular in the extent of the results, both theoretical and experimental, we present here. This paper was developed in the framework of Grenoble Alpes Data Institute (ANR-15-IDEX-02).

\section{Supplement}

The supplementary material Benhenni, Girard and Louhichi (2021) gives first, in its section A.1, the proof of Lemma 2.1. Next, it gives complementary proofs of Lemmas 4.1, 4.2, 4.3, and 4.5, of Propositions 4.3 and 4.5, and of Theorem 3.1. Moreover, some other important probabilistic properties for MDS are stated in Appendices B and C. More precisely, Appendix B gives the central limit theorem for MDS; Appendix $\mathrm{C}$ gives and proves some ingredients for MDS used throughout the proofs of the main results (such as Marcinkiewicz-Zygmund type inequalities or maximal bounds for weighted sums of MDS or quadratic form of MDS).

\section{References}

Akaike, H. (1970). Statistical predictor information. Annals of the Institute of Statistical Mathematics, 22, 203-217. 
Andrew, D. W. K. and Pollard, D. (1994). An introduction to functional central limit theorems for dependent stochastic processes. Int. Stat. Rev. 62, 119-132.

Benhenni, K. and Degras, D. (2014). Local polynomial estimation of the mean function and its derivatives based on functional data and regular designs. ESAIM: Probability and Statistics, EDP Sciences, 18, 881-899.

Benhenni, K. Girard, D. and Louhichi, S. (2021). Supplement to "On bandwidth selection problems in nonparametric trend estimation under martingale difference errors". The DOI will be added by the typesetter.

Available at https://hal .archives-ouvertes.fr/hal-02514827

Burkholder, D. L. (1988). Sharp inequalities for martingales and stochastic integrals. Astérisque 157-158, 75-94.

Craven, P. and Wahba, G. (1979). Smoothing noisy data with spline functions. Numerische Mathematik, 31, 377-403.

Engle, R. F. (1982). Autoregressive Conditional Heteroscedasticity with Estimates of the Variance of United Kingdom Inflation. Econometrica 50-4, 987-1007.

Doukhan, P. and Louhichi, S. (1999). A new weak dependence condition and applications to moment inequalities. Stochastic Process. Appl., 84, 2, 313-342.

Fan, J and Gijbels, I. (1999). Local polynomial modeling and its applications. Monographs on Statistics and Applied Probability, 66, Chapman \& Hall, London.

Francisco-Fernandez, M., Opsomer, J. and Vilar-Fernandez, J.M. (2004). Plug-in bandwidth selector for local polynomial regression estimator with correlated errors. J. Nonparametr. Stat., 16, 127-151.

Gasser, T. and Muller, H.G. (1979). Kernel estimation of regression functions, in Smoothing Techniques in Curve Estimation. Lecture Notes in Mathematics 757, 23-68.

Girard, D.A. (1998). Asymptotic comparison of (partial) cross-validation, GCV and randomized GCV in non-parametric regression. Ann. Stat., 26, 315-334. 
Han, Ch. and Gu, Ch. (2008). Optimal Smoothing with Correlated Data. Sankhya: The Indian Journal of Statistics, Series A, 70, 1, 38-72.

Härdle, W., Hall, P. and Marron, J.S. (1988). How far are automatically chosen regression smoothing parameters from their optimum? Journal of the American Statistical Association 83, 86-95.

Hall, P., Lahiri, S. N. and Polzehl, J. (1995). On bandwidth choice in nonparametric regression with both short and long-range dependent errors. Ann. Statist. 23-6, 1921-1936.

Hastie, T., Tibshirani, R. and Friedman, J. (2009). The Elements of Statistical Learning: Data Mining, Inference, and Prediction. Springer.

Lindner, A.M. (2009). Stationarity, Mixing, Distributional Properties and Moments of GARCH(p, q)-Processes. In: Mikosch T., Kreib JP., Davis R., Andersen T. (eds) Handbook of Financial Time Series. Springer, Berlin, Heidelberg.

Louhichi, S. (2000). Weak convergence for empirical processes of associated sequences. Ann. Inst. Henri Poincaré Probabilités et Statistiques, 36, 5, $547-567$.

Mallows, C. L. (1973). Some comments on Cp. Technometrics 15, 661-675.

McLeish, D. L. (1974). Dependent Central Limit Theorems and Invariance Principles. Ann. Probab. 2, 4, 620-628.

Peligrad, M., Utev, S. and Wu, W. B. (2007). A maximal Lp-inequality for stationary sequences and its applications. Proc. Am. Math. Soc. 135, 541550 .

Pollard, D. (1990). Empirical Processes: Theory and Applications. NSFCBMS Regional Conference Series in Probability and Statistics, Institute of Mathematical Statistics, Hayward, CA.

Rice, J. (1984). Bandwidth choice for nonparametric regression. Annals of Statistics 12, 1215-1230.

Rio, E. (1993). Covariance inequalities for strongly mixing processes. Ann. Inst. H. Poincaré Probab. Statist., 29 587-597. 
Rio, E. (2017). Asymptotic theory of weakly dependent random processes. Probability Theory and Stochastic Modelling, 80. Springer.

Shibata, R. (1981). An optimal selection of regression variables. Biometrika, 68, $45-54$.

Nadaraya, E. A. (1964). On Estimating Regression. Theory of Probability and Its Applications. 9 (1), 141-2.

Watson, G. S. (1964). Smooth regression analysis. Sankhya: The Indian Journal of Statistics, Series A. 26, 359-372.

Wang, Y. (1998). Smoothing Spline Models with Correlated Random Errors. Journal of the American Statistical Association, 93, 441, 41-348.

Welsh, A.H., Lin, X. and Carroll, R.J., 2002. Marginal longitudinal nonparametric regression: locality and efficiency of spline and kernel methods. Journal of the American Statistical Association, 97, 458, 482-493. 